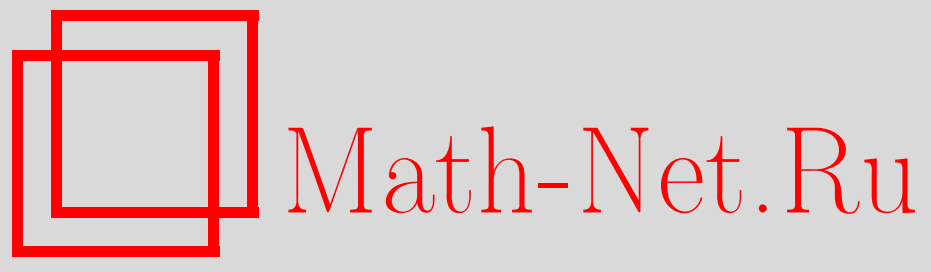

А. В. Беляев, Об общем решении задачи о движении тяжелого твердого тела в случае Гесса, Матем. сб., 2015, том 206, номер 5, 5-34

DOI: https://doi.org/10.4213/sm8335

Использование Общероссийского математического портала Math-Net.Ru подразумевает, что вы прочитали и согласны с пользовательским соглашением http://www . mathnet.ru/rus/agreement

Параметры загрузки:

IP : 54.162 .127 .20

26 апреля 2023 г., 14:42:12

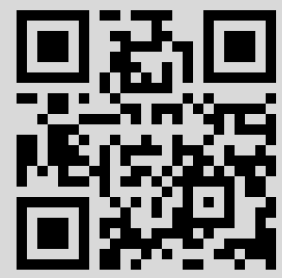




\section{А. В. Беляев \\ Об общем решении задачи о движении тяжелого твердого тела в случае Гесса}

Решение уравнений Эйлера-Пуассона в случае Гесса представлено набором особых точек решения вместе с асимптотикой решения в этих точках. Указан полный список однозначных и конечнозначных решений случая Гесса. Получено представление для предельных периодических решений и найдено точное условие, при котором такие решения существуют.

Библиография: 25 названий.

Ключевые слова: первый интеграл, случай Гесса уравнений Эйлера-Пуассона, асимптотика решений, особые точки решений, аналитические функции.

DOI: $10.4213 / \operatorname{sm} 8335$

\section{§ 1. Введение}

Случай Гесса (см. [1]) задачи о движении тяжелого твердого тела интересен тем, что, несмотря на наличие четвертого интеграла для классических уравнений Эйлера-Пуассона, его решения в квадратурах не удалось получить никому. Тем не менее и без явного решения их свойства исследованы достаточно полно. Геометрическое истолкование искомого движения было дано Е. Н. Жуковским в [2], а П. А. Некрасов и Б. К. Млодзеевский в [3], [4] доказали, что при некоторых ограничениях решения могут быть асимптотическими. Представляет интерес также тот факт, что случай Гесса был переоткрыт Г. Г. Аппельротом (см. [5]), использовавшим идею С. В. Ковалевской (см. [6]) исследования на однозначность особых точек решений уравнений Эйлера-Пуассона. Отметим также, что П. А. Некрасовым в [7] показано, что решения в случае Гесса являются, вообще говоря, неоднозначными.

В настоящее время классическая задача о движении тяжелого твердого тела в первую очередь представляет интерес в связи тематикой интегрирования гамильтоновых систем методом LA-пары с параметром (см. монографию [8]). Для гамильтоновых систем на алгебрах Ли этот метод был представлен в работах [9]-[12].

K случаю Гесса метод LA-пары с параметром был применен в работе В. Драговича и Б. Гаджича [13]. Естественно, что этот метод не мог дать общего решения, так как оно не выражается в $\theta$-функциях. Но все же были получены частные периодические решения и вдобавок сам факт существования LA-пары с параметром для, вообще говоря, неинтегрируемой задачи является весьма интересным. 
Из современных работ, посвященных исследованию классического случая Гесса, необходимо также отметить статьи П. Любовецки и Х. Золадека [14], [15]. В этих работах исследуются топологические свойства потока на фазовом пространстве задачи Гесса и ее возмущения.

Итак, случай Гесса, как и общая задача о движении тяжелого твердого тела, является актуальным и сегодня.

Неоднозначность решений в случае Гесса и отсутствие интегрируемости по Лиувиллю (см. [16]) существенно усложняет исследование задачи, но, тем не менее, достаточно полное описание аналитических свойств решений возможно.

В настоящей статье мы рассматриваем аналитические свойства решений задачи Гесса, т.е. используем метод исследования, отличающийся от методов работ [13]-[15]. Мы представляем решения уравнений Эйлера-Пуассона в случае Гесса как аналитические функции времени с заданными асимптотиками в окрестностях заданных особых точек. Этот результат отличается от результатов классических и современных исследований тем, что в них не ставилась, а значит и не решалась, задача полного описания асимптотик всех особых точек данного решения вместе с указанием координат самих особых точек на комплексной временной плоскости. При этом необходимо отметить, что асимптотика особых точек имеет свободные параметры, однако для фиксированного решения они свободны лишь в одной заранее выбранной особой точке, а в остальных не являются свободными и связаны некоторой зависимостью. Эта зависимость может быть описана в терминах отображения, которое мы называем параметрическим отображением последования (см. [17]).

Мы полагаем, что можно считать, что найдено аналитическое решение дифференциальных уравнений, в данном случае задачи Гесса, если для данного решения или класса решений указаны координаты особых точек, асимптотики всех особых точек и соотношения, связывающие свободные параметры особых точек. Отметим, что представление такого же типа с тождественным отображением последования имеют и классические эллиптические функции (см. [18]), и это представление позволяет получить все известные как локальные, так и глобальные свойства. Хотя классическим решением дифференциального уравнения считается решение в квадратурах, оно не всегда эффективно с точки зрения исследования свойств полученного решения, если интегралы явно не берутся.

Чтобы показать содержательность полученного в статье представления решения задачи Гесса, мы доказываем полноту списка известных однозначных решений, а также доказываем, что конечнозначных решений, не являющихся однозначными, нет.

В статье мы используем представление решений задачи Гесса с помощью уравнения Риккати, позволяющее получить точную оценку для границы предельных периодических решений.

Выражаем искреннюю признательность А. Т. Фоменко за поддержку. 


\section{§ 2. Формулировка основных результатов}

В соответствии с изложенным подходом основной результат статьи сформулируем следующим образом.

Теорема 1. Решение уравнений Эйлера-Пуассона

$$
\left\{\begin{array}{l}
A \dot{p}=A p \times p+\gamma \times r, \\
\dot{\gamma}=\gamma \times p,
\end{array}\right.
$$

где

$$
\begin{gathered}
p=\left(p_{1}, p_{2}, p_{3}\right) \in \mathbb{C}^{3}, \quad \gamma=\left(\gamma_{1}, \gamma_{2}, \gamma_{3}\right) \in \mathbb{C}^{3}, \\
A=\operatorname{diag}\left(A_{1}, A_{2}, A_{3}\right), \quad A: \mathbb{R}^{3} \rightarrow \mathbb{R}^{3}, \quad r=\left(r_{1}, r_{2}, r_{3}\right) \in \mathbb{R}^{3},
\end{gathered}
$$

в случае Гесса, задаваемого условиями

$$
\begin{gathered}
A_{1} B_{23} r_{1}^{2}=A_{2} B_{31} r_{2}^{2}, \quad r_{3}=0 \\
\mathscr{I}=A_{1} r_{1} p_{1}+A_{2} r_{2} p_{2}=0
\end{gathered}
$$

(индексы можно ииклически переставлять), имеет следующее представление, выражающее переменные $p_{i}, \gamma_{i}, i=1,2,3$, через функиии $\mathscr{F}, \varphi$ :

$$
\left\{\begin{aligned}
p_{i} & =k_{i} \sqrt{\mathscr{F}} \frac{\varphi}{1+\varphi^{2}}, \quad i=1,2 \\
p_{3} & =k_{3} \sqrt{\mathscr{F}} \frac{1-\varphi^{2}}{1+\varphi^{2}} \\
\gamma_{1} & =\frac{1}{\mathscr{R}}\left(r_{1}(\mathscr{H}-F)+r_{2}\left(A_{3} \dot{p}_{3}-B_{12} p_{1} p_{2}\right)\right) \\
\gamma_{2} & =\frac{1}{\mathscr{R}}\left(r_{2}(\mathscr{H}-\mathscr{F})-r_{1}\left(A_{3} \dot{p}_{3}-B_{12} p_{1} p_{2}\right)\right) \\
\gamma_{3} & =\frac{1}{r_{2}}\left(B_{23} p_{2} p_{3}-A_{1} \dot{p}_{1}\right)
\end{aligned}\right.
$$

здесь

$$
\begin{gathered}
k_{2}=-\frac{A_{1} r_{1}}{A_{2} r_{2}} k_{1}, \quad k_{1}=2 \sqrt{\frac{2 B_{23}}{-B_{12} A_{1}}}, \quad k_{3}=\sqrt{\frac{2}{A_{3}}}, \\
A_{1}<A_{3}<A_{2}, \quad B_{i j}=A_{i}-A_{j} .
\end{gathered}
$$

Функиия Я્F является в общем случае эллиптической и задается дифференциальным уравнением

$$
A_{3}^{2}(\dot{\mathscr{F}})^{2}=2 A_{3} \mathscr{T} \mathscr{R} \mathscr{F}-2 A_{3} \mathscr{F}(\mathscr{F}-\mathscr{H})^{2}-\mathscr{M}^{2} \mathscr{R}=A_{3}^{2} \mathscr{P}_{3}(\mathscr{F})
$$

здесъ $\mathscr{H}=\frac{1}{2}\langle A p, p\rangle+\langle\gamma, r\rangle, \mathscr{M}=\langle A p, \gamma\rangle, \mathscr{T}=\langle\gamma, \gamma\rangle,-$ первые интеграль исходной системь уравнений Эйлера-Пуассона, $\mathscr{R}=\langle r, r\rangle,\langle x, y\rangle=\sum_{i=1}^{3} x_{i} y_{i}-$ $\mathbb{C}$-скалярное произведение в $\mathbb{C}^{3}$.

Если корни полинома $\mathscr{P}_{3}(\mathscr{F})$ обозначить $x_{1}<x_{2}<x_{3}$, то

$$
\mathscr{F}(t)=a \operatorname{dn}^{2}(\omega t, k)+b,
$$


әде

$$
b=x_{1}, \quad a=x_{3}-x_{1}, \quad k^{2}=\frac{x_{3}-x_{2}}{x_{3}-x_{1}}, \quad \omega^{2}=\frac{x_{3}-x_{1}}{2 A_{3}} .
$$

Кроме того,

$2 \mathscr{M} \sqrt{\mathscr{R}}=x_{1} x_{2} x_{3}, \quad 2 \mathscr{H}=x_{1}+x_{2}+x_{3}, \quad \mathscr{H}^{2}-\mathscr{R} \mathscr{T}=x_{1} x_{2}+x_{2} x_{3}+x_{3} x_{1}$.

Функиия ч задается дифберенциальным уравнением Риккати

$$
\dot{\varphi}=\nu \sqrt{\mathscr{F}} \varphi-\frac{\kappa}{\mathscr{F}}\left(1+\varphi^{2}\right), \quad \nu=-\frac{\sqrt{2 B_{23} B_{31}}}{\sqrt{A_{1} A_{2} A_{3}}}, \quad \kappa=\frac{\mathscr{M} \sqrt{R}}{4 A_{3}} .
$$

Нули и полюсы функции Ғ являются особыми точками функиии ч, в окрестностях которых ее асимптотика имеет вид:

- в окрестности одной из сопряженных точек $\mathscr{F}=0-$

$$
\varphi=-i+c t^{1 / 2}+\frac{i c^{2}}{2} t-\left(\frac{i c \sigma}{16 \kappa}+\frac{c^{3}}{4}+2 \nu i \sqrt{\kappa i}\right) t^{3 / 2}+\cdots,
$$

где $c \in \mathbb{C}$ - свободный параметр, определяющий решение из однопараметрического семейства решений на двумерном торе, задаваемом интегралами $\mathscr{H}$, $\mathscr{M}, \mathscr{T}, \mathscr{I}$, либо

$$
\varphi=i+\nu i \sqrt{\kappa i} t^{3 / 2}+\cdots
$$

- в окрестности точки $\mathscr{F}=\infty-$

$$
\varphi=c t^{\lambda \nu}+\cdots+\frac{\kappa t^{3}}{2 A_{3}(3-\lambda \nu)}+\cdots
$$

где с $\in \mathbb{C}$ - свободный параметр, определяющий решение из однопараметрического семейства решений на двумерном торе, задаваемом интегралами $\mathscr{H}$, $\mathscr{M}, \mathscr{T}, \mathscr{I}$, либо

$$
\varphi=-\frac{2 A_{3}(3+\lambda \nu)}{\kappa t^{3}}-\frac{2 H(3+\nu \lambda)\left(A_{3} \nu-(3+\nu \lambda) \lambda\right)}{3 \kappa \lambda(5+\lambda \nu) t}+\cdots,
$$

где $\lambda= \pm i \sqrt{2 A_{3}}$.

В пределах двух соседних параллелограммов периодов эллиптической функиии $\mathscr{F}$ свободные параметры асимптотик с различных особых точек связаны дробно-линейными преобразованиями, которье, вообще говоря, не коммутируют друг с другом в силу неоднозначности решений. Дробно-линейнье преобразования, связывающие свободные параметры асимптотик произвольных особых точек $\mathscr{F}=\infty$, представляют собой композиции следующих преобразований:

$$
\begin{aligned}
& \Phi_{\tau_{1}+}: c \rightarrow \frac{a_{11} c+a_{12}}{a_{21} c+a_{22}}, \quad \operatorname{det}\left(\begin{array}{ll}
a_{11} & a_{12} \\
a_{21} & a_{22}
\end{array}\right)=1, \\
& \Phi_{\tau_{1}-}: c \rightarrow-\frac{a_{22} c-a_{21}}{a_{12} c-a_{11}}
\end{aligned}
$$




$$
\begin{aligned}
& \Phi_{\tau_{2}+}: c \rightarrow \frac{b_{11} c+b_{12}}{b_{21} c+b_{22}}, \quad \operatorname{det}\left(\begin{array}{ll}
b_{11} & b_{12} \\
b_{21} & b_{22}
\end{array}\right)=1, \\
& \Phi_{\tau_{2}-}: c \rightarrow-\frac{b_{22} c-b_{21}}{b_{12} c-b_{11}} .
\end{aligned}
$$

В формулах (2.13)-(2.16) $\tau_{1}$ - вещественный, $i \tau_{2}-$ мнимый периоды функuиu $\mathscr{F}$,

$$
\Phi_{\tau_{1} \pm}: s_{ \pm} \rightarrow s_{ \pm}, \quad \Phi_{\tau_{2} \pm}: s_{\mp} \rightarrow s_{ \pm}
$$

- параметрические отображения последования вдоль вещественной и мнимой осей соответственно; $s_{ \pm}-$особые точки $\mathscr{F}=\infty$ со значением параметра $\lambda= \pm i \sqrt{2 A_{3}}$,

$$
\begin{gathered}
a_{11}=\delta^{2} a_{22} \in \mathbb{R}, \quad a_{12}=-\delta^{2} \bar{a}_{21}, \quad \delta^{2}=\exp \left(2 \pi \nu \sqrt{2 A_{3}}\right), \\
b_{22}=\bar{b}_{11}, \quad b_{21}=-\delta^{2} b_{12} \in i \mathbb{R}, \\
a_{11}-a_{22}=2 \operatorname{Re}\left(a_{21} \frac{b_{11}}{b_{21}}\right) .
\end{gathered}
$$

Параметры $a_{12} \in \mathbb{C},\left|b_{11}\right| \in \mathbb{R}$ с учетом зависимостей (2.13), (2.15) и (2.17)-(2.19) можно считать свободнъми, определяющими $\mathbb{R}$-трехпараметрическое семейство вещественных инвариантных торов задачи случая Гесса, точно так же, как и параметры $x_{i}, i=1,2,3$, или $\mathscr{H}, \mathscr{M}, \mathscr{T}$ при фиксированных параметрах твердого тела $A_{i}, r_{i}, i=1,2,3$.

В случае $\left|a_{11}+a_{22}\right|>2$ решения задачи Гесса предельно-периодичны, в случае $\left|a_{11}+a_{22}\right|<2$ - квазипериодичны (см. [19]).

Доказательство теоремы 1 составляет основное содержание статьи, которую мы разбиваем на параграфы следующим образом. В $\S 3$ изложены классические результаты, характеризующие случай Гесса, а также доказываются формулы (2.4)-(2.8). При этом уравнения (2.1) случая Гесса сводятся к уравнению Риккати и к системе линейных уравнений. Сам факт возможности такого сведения является хорошо известным классическим результатом (см. [4]), однако виды уравнений Риккати и соответственно линейных систем могут быть самыми различными, поэтому мы приводим соответствующий вывод. В $₫ 4$ доказываются формулы (2.9)-(2.12), а также приводятся асимптотики особых точек решений этого случая. В $\$ 5$ исследуется зависимость между параметрами асимптотик особых точек задачи Гесса, доказываются формулы (2.13)-(2.18) и, тем самым, завершается доказательство теоремы 1.

В $\S 6$ в качестве приложения теоремы 1 мы доказываем теорему 2 о полном списке однозначных решений случая Гесса задачи о движении тяжелого твердого тела и предложение 9 о полном списке конечнозначных решений. В $\S 7$ доказывается теорема 3 о квазипериодических и предельно-периодических решениях случая Гесса. Наконец, в $\S 8$ в теореме 4 дается представление предельнопериодических решений и найдено точное условие, при выполнении которого эти решения существуют. 


\section{§ 3. Уравнения Эйлера-Пуассона в случае Гесса}

В этом параграфе доказываются формулы (2.4)-(2.8) теоремы 1.

Классические уравнения Эйлера-Пуассона (2.1), описывающие движение тяжелого твердого тела, в случае Гесса, т.е. при условии (2.2)

$$
A_{1} B_{23} r_{1}^{2}=A_{2} B_{31} r_{2}^{2}, \quad r_{3}=0,
$$

на параметры твердого тела имеют дополнительный четвертый интеграл:

$$
\mathscr{I}=\langle A p, r\rangle=0,
$$

кроме общих первых интегралов

$$
\begin{gathered}
\mathscr{H}=\frac{1}{2}\langle A p, p\rangle+\langle\gamma, r\rangle, \\
\mathscr{M}=\langle A p, \gamma\rangle, \\
\mathscr{T}=\langle\gamma, \gamma\rangle,
\end{gathered}
$$

где $\langle x, y\rangle=\sum_{i=1}^{3} x_{i} y_{i}-\mathbb{C}$-скалярное произведение в $\mathbb{C}^{3}$.

Заметим, что четвертый интеграл сохраняется только на нулевой поверхности уровня самого интеграла и именно эта его особенность приводит к целому ряду необычных свойств случая Гесса.

Далее для определенности будем считать, что

$$
A_{1}<A_{3}<A_{2} \text {. }
$$

Введем функции $\mathscr{F}=\frac{1}{2}\langle A p, p\rangle, \mathscr{G}=\frac{1}{2}\langle A p, A p\rangle$, для которых

$$
\begin{gathered}
\dot{\mathscr{F}}=\langle A p \times p, p\rangle+\langle\gamma \times r, p\rangle=\langle\gamma \times r, p\rangle, \\
\dot{\mathscr{G}}=\langle A p \times p, A p\rangle+\langle\gamma \times r, A p\rangle=\langle\gamma \times r, A p\rangle .
\end{gathered}
$$

Используя соотношения

$$
\begin{gathered}
\langle a, b\rangle\langle c, d\rangle=\langle a, c\rangle\langle b, d\rangle+\langle a \times d, b \times c\rangle, \\
(a \times b) \times c=\langle a, c\rangle b-\langle b, c\rangle a,
\end{gathered}
$$

получим

$$
\begin{aligned}
\dot{\mathscr{F}} \mathscr{G} & =\langle A p, A p\rangle\langle\gamma \times r, p\rangle=\mathscr{F} \dot{\mathscr{G}}+\langle A p \times(\gamma \times r), A p \times p\rangle \\
& =\mathscr{F} \dot{\mathscr{G}}-\mathscr{M}\langle A p \times p, r\rangle=\mathscr{F} \dot{\mathscr{G}}-\mathscr{M}\langle A p, r\rangle=\mathscr{F} \dot{\mathscr{G}} .
\end{aligned}
$$

Из последнего соотношения следует, что $\mathscr{G} / \mathscr{F}=$ const. Найдем значение этой константы:

$$
\begin{aligned}
A_{1} p_{1} r_{1}+A_{2} p_{2} r_{2}=0 & \Longrightarrow p_{2}=-\frac{A_{1} p_{1} r_{1}}{A_{2} r_{2}} \Longrightarrow p_{2}^{2}=\frac{A_{1}^{2} p_{1}^{2} r_{1}^{2}}{A_{2}^{2} r_{2}^{2}} \\
& \Longrightarrow\left\{\begin{array}{l}
2 \mathscr{G}=A_{1}^{2} p_{1}^{2}\left(1+\frac{r_{1}^{2}}{r_{2}^{2}}\right)+A_{3}^{2} p_{3}^{2} \\
2 \mathscr{F}=A_{1} p_{1}^{2}\left(1+\frac{A_{1} r_{1}^{2}}{A_{2} r_{2}^{2}}\right)+A_{3} p_{3}^{2}
\end{array}\right.
\end{aligned}
$$


Используя соотношение $(2.2)$, получим

$$
\left\{\begin{array}{l}
2 \mathscr{G} B_{23}=A_{1} p_{1}^{2}\left(A_{1} B_{23}+A_{2} B_{31}\right)+A_{3}^{2} B_{23} p_{3}^{2}, \\
2 \mathscr{F} B_{23}=A_{1} p_{1}^{2}\left(B_{23}+B_{31}\right)+A_{3} B_{23} p_{3}^{2},
\end{array}\right.
$$

откуда следует, что $\mathscr{G}=A_{3} \mathscr{F}$.

Введем обозначение $\mathscr{R}=\langle r, r\rangle$ и воспользуемся соотношениями (3.5), (3.6):

$$
\begin{aligned}
(\dot{\mathscr{G}})^{2}= & \langle A p, \gamma \times r\rangle\langle A p, \gamma \times r\rangle=2 \mathscr{G}\langle\gamma \times r, \gamma \times r\rangle \\
& \quad-\langle A p \times(\gamma \times r), A p \times(\gamma \times r)\rangle=2 \mathscr{G}\langle(\gamma \times r) \times \gamma, r\rangle-\mathscr{M}^{2} \mathscr{R} \\
= & 2 \mathscr{G}\left(\mathscr{T} \mathscr{R}-\langle\gamma, r\rangle^{2}\right)-\mathscr{M}^{2} \mathscr{R}=2 \mathscr{G} \mathscr{T} \mathscr{R}-2 \mathscr{G}(\mathscr{F}-\mathscr{H})^{2}-\mathscr{M}^{2} \mathscr{R} .
\end{aligned}
$$

Итак, мы можем найти функции $\mathscr{F}, \mathscr{G}$ из уравнения (2.5)

$$
A_{3}^{2}(\dot{\mathscr{F}})^{2}=2 A_{3} \mathscr{T} \mathscr{R} \mathscr{F}-2 A_{3} \mathscr{F}(\mathscr{F}-\mathscr{H})^{2}-\mathscr{M}^{2} \mathscr{R}
$$

Рассмотрим следующее представление для $p(t)$ (см. (2.4)):

$$
\left\{\begin{array}{l}
p_{i}=k_{i} \sqrt{\mathscr{F}} \frac{\varphi}{1+\varphi^{2}}, \quad i=1,2, \\
p_{3}=k_{3} \sqrt{\mathscr{F}} \frac{1-\varphi^{2}}{1+\varphi^{2}}
\end{array}\right.
$$

где

$$
k_{2}=-\frac{A_{1} r_{1}}{A_{2} r_{2}} k_{1}, \quad k_{1}=2 \sqrt{\frac{2 B_{23}}{-B_{12} A_{1}}}, \quad k_{3}=\sqrt{\frac{2}{A_{3}}} .
$$

Представление мы выбираем таким образом, чтобы тождественно выполнялись условия $\langle A p, r\rangle=0,\langle A p, p\rangle=2 \mathscr{F}$. Естественно, что при этом общее количество свободных переменных уменьшается на 2.

Используя имеющиеся интегралы, получим дифференциальное уравнение для $\varphi$ :

$$
A_{1} \dot{p}_{1}=B_{23} p_{2} p_{3}-r_{2} \gamma_{3} \quad \Longrightarrow \quad \gamma_{3}=\frac{1}{r_{2}}\left(B_{23} p_{2} p_{3}-A_{1} \dot{p}_{1}\right) .
$$

Из системы

$$
\left\{\begin{array}{l}
\gamma_{1} r_{1}+\gamma_{2} r_{2}=\mathscr{H}-\mathscr{F} \\
\gamma_{1} r_{2}-\gamma_{2} r_{1}=A_{3} \dot{p}_{3}-B_{12} p_{1} p_{2}
\end{array}\right.
$$

найдем $\gamma_{1}$ и $\gamma_{2}($ см. (2.4)):

$$
\left\{\begin{array}{l}
\gamma_{1}=\frac{1}{\mathscr{R}}\left(r_{1}(\mathscr{H}-\mathscr{F})+r_{2}\left(A_{3} \dot{p}_{3}-B_{12} p_{1} p_{2}\right)\right), \\
\gamma_{2}=\frac{1}{\mathscr{R}}\left(r_{2}(\mathscr{H}-\mathscr{F})-r_{1}\left(A_{3} \dot{p}_{3}-B_{12} p_{1} p_{2}\right)\right) .
\end{array}\right.
$$

Заметим, что

$$
A_{1} p_{1} r_{2}-A_{2} p_{2} r_{1}=A_{1} p_{1} r_{2}+A_{2} r_{1} \frac{A_{1} r_{1}}{A_{2} r_{2}} p_{1}=\frac{A_{1} p_{1}}{r_{2}} \mathscr{R},
$$


и подставим имеющиеся представления переменных $p_{i}, \gamma_{i}$ в интеграл момента $\mathscr{M}$. Мы получим

$$
\begin{aligned}
\mathscr{M} & =\frac{A_{1} p_{1}}{r_{2}}\left(A_{3} \dot{p}_{3}-B_{12} p_{1} p_{2}\right)+\frac{A_{3} p_{3}}{r_{2}}\left(-A_{1} \dot{p}_{1}+B_{23} p_{2} p_{3}\right) \\
& =\frac{A_{1} A_{3}}{r_{2}}\left(p_{1} \dot{p}_{3}-\dot{p}_{1} p_{3}\right)+\frac{p_{2}}{r_{2}}\left(A_{3} B_{23} p_{3}^{2}-A_{1} B_{12} p_{1}^{2}\right) \\
& =\frac{A_{1} A_{3}}{r_{2}}\left(\frac{p_{3}}{p_{1}}\right) p_{1}^{2}+\frac{2 B_{23}}{r_{2}} \mathscr{F} p_{2} \\
& =\frac{A_{1} A_{3}}{r_{2}} k_{1} k_{3} \mathscr{F}\left(\frac{1-\varphi^{2}}{\varphi}\right) \cdot \frac{\varphi^{2}}{\left(1+\varphi^{2}\right)^{2}}+\frac{2 B_{23}}{r_{2}} k_{2} \mathscr{F}^{3 / 2} \frac{\varphi}{1+\varphi^{2}} .
\end{aligned}
$$

Наконец, поскольку

$$
\left(\frac{1-\varphi^{2}}{\varphi}\right)=-\dot{\varphi} \frac{1+\varphi^{2}}{\varphi^{2}}
$$

мы получаем (см. (2.8))

$$
\dot{\varphi}=\nu \sqrt{\mathscr{F}} \varphi-\frac{\kappa}{\mathscr{F}}\left(1+\varphi^{2}\right), \quad \nu=-\frac{\sqrt{2 B_{23} B_{31}}}{\sqrt{A_{1} A_{2} A_{3}}}, \quad \kappa=\frac{\mathscr{M} \sqrt{R}}{4 A_{3}} .
$$

ЗАмЕчАниЕ 1 (см. [1]). Если $\mathscr{M}=0$, то $\kappa=0$ и уравнения (3.7) явно интегрируются.

Полагая $\varphi=\theta_{1} / \theta_{2}$, получим линейную систему для $\theta=\left(\theta_{1}, \theta_{2}\right)$ :

$$
\begin{aligned}
& \frac{1}{\theta_{2}^{2}}\left(\dot{\theta}_{1} \theta_{2}-\theta_{1} \dot{\theta}_{2}\right)=\nu \sqrt{\mathscr{F}} \frac{\theta_{1}}{\theta_{2}}-\frac{\kappa}{\mathscr{F}}\left(1+\frac{\theta_{1}^{2}}{\theta_{2}^{2}}\right) \\
& \Longrightarrow \quad \dot{\theta}_{1} \theta_{2}-\theta_{1} \dot{\theta}_{2}=\nu \sqrt{\mathscr{F}} \theta_{1} \theta_{2}-\frac{\kappa}{\mathscr{F}}\left(\theta_{1}^{2}+\theta_{2}^{2}\right) \\
& =\left(\frac{\nu}{2} \sqrt{\mathscr{F}} \theta_{1}-\frac{\kappa}{\mathscr{F}} \theta_{2}\right) \theta_{2}-\left(-\frac{\nu}{2} \sqrt{\mathscr{F}} \theta_{2}+\frac{\kappa}{\mathscr{F}} \theta_{1}\right) \theta_{1} \\
& \Longrightarrow \quad\left\{\begin{array}{l}
\dot{\theta}_{1}=\frac{\nu}{2} \sqrt{\mathscr{F}} \theta_{1}-\frac{\kappa}{\mathscr{F}} \theta_{2}, \\
\dot{\theta}_{2}=\frac{\kappa}{\mathscr{F}} \theta_{1}-\frac{\nu}{2} \sqrt{\mathscr{F}} \theta_{2} .
\end{array}\right.
\end{aligned}
$$

ЗАмечАниЕ 2. В работе [7] П.А. Некрасов свел динамические уравнения в случае Гесса к уравнению Риккати, для которого записал линейную систему дифференциальных уравнений. Однако мы предпочитаем не пользоваться этим результатом, поскольку неизвестная величина этой линейной системы связана с исходными переменными $(p, \gamma)$ дифференциальными соотношениями.

Как следует из классических результатов (см. [18]), функцию $\mathscr{F}$, удовлетворяющую дифференциальному уравнению (2.5), можно представить в виде (2.6):

$$
\mathscr{F}(t)=a \operatorname{dn}^{2}(\omega t, k)+b .
$$

Подставив это представление в дифференциальное уравнение (2.5) и выразив все эллиптические функции через $\operatorname{dn}^{2}(\omega t, k)$, приравняем коэффициенты 
при $\mathrm{dn}^{2 n}(\omega t, k), n=0, \ldots, 3$, к нулю. Мы получим следующие соотношения:

$$
\begin{gathered}
2 A_{3} \mathscr{T} \mathscr{R} b-2 A_{3} b(b-\mathscr{H})^{2}-\mathscr{M}^{2} \mathscr{R}=0, \\
a^{2}+3 a b-2 \mathscr{H} a+3 b^{2}-4 \mathscr{H} b+\mathscr{H}^{2}-\mathscr{R} \mathscr{T}=0, \\
2 A_{3} \omega^{2}-a=0, \quad a k^{2}-2 a-3 b+2 \mathscr{H}=0 .
\end{gathered}
$$

Хотя параметр $b$ удовлетворяет уравнению третьей степени (кстати, отметим, совпадающему с $\left.\mathscr{P}_{3}(\mathscr{F})=0\right)$, из трех корней только один подходит для рассматриваемого представления. В самом деле, дифференциальное уравнение (2.5) задает движение, только если

$$
\mathscr{F}=\frac{1}{2}\langle A p, p\rangle>0, \quad A_{3}^{2}(\dot{\mathscr{F}})^{2}=2 A_{3} \mathscr{T} \mathscr{R} \mathscr{F}-2 A_{3} \mathscr{F}(\mathscr{F}-\mathscr{H})^{2}-\mathscr{M}^{2} \mathscr{R}>0 .
$$

Но при этом полином третьей степени от $\mathscr{F}$ имеет один отрицательный корень $x_{1}$ и отрицательную производную при больших степенях $\mathscr{F}$. Это значит, что в рассматриваемом случае два оставшихся корня положительны: $x_{3}>$ $x_{2}>0>x_{1}$, и значения $\mathscr{F}$ находятся между ними. Иначе говоря, $\mathscr{F}_{\min }=x_{2}$, a $\mathscr{F}_{\max }=x_{3}$. Учитывая свойства функции $\operatorname{dn}^{2}(\omega t, k)$, получаем

$$
\begin{aligned}
\mathscr{F}_{\text {max }} & =a \operatorname{dn}^{2}(\omega t, k)_{\text {max }}+x_{i}=a+x_{i}=x_{3}, \\
\mathscr{F}_{\text {min }} & =a \operatorname{dn}^{2}(\omega t, k)_{\text {min }}+x_{i}=a\left(1-k^{2}\right)+x_{i}=x_{2},
\end{aligned}
$$

где $0<k<1$. Так как $a>0$, то, очевидно, $x_{i}=x_{1}$.

Итак, мы имеем представления для $a, b, k^{2}$, выраженные через корни полинома

$$
\mathscr{P}_{3}(x)=x^{3}-2 \mathscr{H} x^{2}+\left(\mathscr{H}^{2}-\mathscr{R} \mathscr{T}\right) x+8 A_{3} \kappa=0 .
$$

Остается выразить $\omega^{2}$ через $x_{i}$ и убедиться, что полученные представления не противоречат оставшимся соотношениям.

ПРЕДЛОЖЕНИЕ 1. При обходе вокруг особой точки $\mathscr{F}=0$ функиия $\varphi$ изменяется так, что

$$
\varphi_{+} \varphi_{-}=-1
$$

¿əe

$$
\varphi_{+}=\left.\varphi_{+}\right|_{\sqrt{\mathscr{F}}>0}, \quad \varphi_{+}=\left.\varphi_{-}\right|_{\sqrt{\mathscr{F}}<0} .
$$

ДокАзАтЕЛЬСтво. В обозначениях теоремы 1 получаем, что

$$
\frac{p_{1}}{p_{3}}=\frac{k_{1}}{k_{3}} \frac{\varphi}{1-\varphi^{2}} .
$$

Положим $k_{3} p_{1} /\left(k_{1} p_{3}\right)=\eta$ и тогда имеем

$$
\begin{gathered}
\eta \varphi^{2}+\varphi-\eta=0, \\
\varphi_{1}=\frac{-1+\sqrt{1+4 \eta^{2}}}{2 \eta}, \quad \varphi_{2}=\frac{-1-\sqrt{1+4 \eta^{2}}}{2 \eta},
\end{gathered}
$$


причем $\varphi_{1} \varphi_{2}=-1$.

Следующее дифференциальное соотношение показывает, что $\varphi_{1}, \varphi_{2}$ удовлетворяют (3.7) на разных листах $\sqrt{\mathscr{F}}$ :

$$
\left(-\frac{1}{\varphi}\right)=\frac{\dot{\varphi}}{\varphi^{2}}=\frac{\nu \sqrt{\mathscr{F}} \varphi-\frac{\kappa}{\mathscr{F}}\left(1+\varphi^{2}\right)}{\varphi^{2}}=-\nu \sqrt{\mathscr{F}}\left(-\frac{1}{\varphi}\right)-\frac{\kappa}{\mathscr{F}}\left(1+\left(-\frac{1}{\varphi}\right)^{2}\right) .
$$

ЗАмЕчАниЕ 3. При переходе от переменной $\varphi$ к исходным переменным $p_{i}, \gamma_{i}$ решения $\varphi_{+}, \varphi_{-}$задают одно и то же решение.

\section{§ 4. Асимптотика особых точек решений уравнений Эйлера-Пуассона в случае Гесса}

В этом параграфе доказываются формулы (2.9)-(2.12) теоремы 1.

Как следует из результатов [20], [21] (см. также [5] и [6]), все особые точки решений уравнений Эйлера-Пуассона, задающие движение тяжелого твердого тела с закрепленной точкой, по своему характеру образуют два класса: $\alpha$ и $\beta$-точки.

Асимптотика $\alpha$-точек решений уравнений Эйлера-Пуассона в случае Гесса имеет вид

$$
\left\{\begin{array}{l}
p(t)=\widetilde{p}^{0} t^{-1}+\alpha_{1} u_{1}+\sum_{i=2}^{4} \alpha_{i} v_{i} t+o(t), \\
\gamma(t)=\alpha_{1} v_{1} t^{-1}+\kappa_{0} v_{1}+\alpha_{4} v \widetilde{p}^{0}+\alpha_{5} v_{-1} t+o(t),
\end{array}\right.
$$

где $\widetilde{p}^{0}$ является решением системы уравнений

$$
\left\{\begin{array}{l}
A \widetilde{p}^{0} \times \widetilde{p}^{0}+A \widetilde{p}^{0}=0 \\
\left\langle A \widetilde{p}^{0}, r\right\rangle=0
\end{array}\right.
$$

и имеет вид

$$
\left( \pm i \sqrt{\frac{A_{2} A_{3}}{-B_{12} B_{31}}}, \pm i \sqrt{\frac{A_{1} A_{3}}{-B_{12} B_{23}}},-\sqrt{\frac{A_{1} A_{2}}{B_{23} B_{31}}}\right) .
$$

Здесь:

$v_{1}, v_{-1}-$ собственные векторы оператора $\xi \rightarrow \widetilde{p}^{0} \times \xi$ с собственными значениями 1 и -1 соответственно (в силу (4.2) $v_{1}=A \widetilde{p}^{0}$ );

$\left\{v_{2}, v_{3}\right\}$ - собственные векторы оператора

$$
\mathbf{D}: \xi \rightarrow A^{-1}\left(A \widetilde{p}^{0} \times \xi+A \xi \times \widetilde{p}^{0}\right)
$$

с собственным значением 1 ;

$v_{4}=-(\mathbf{D}-E)^{-1} A^{-1}\left(\widetilde{p}^{0} \times r\right)$ (в силу вырожденности оператора $\mathbf{D}$ вектор $v_{4}$ определен по модулю пространства $\left.\left\{v_{2}, v_{3}\right\}\right)$;

$$
\begin{aligned}
& u_{1}=-\mathbf{D}^{-1} A^{-1}\left(v_{1} \times r\right) ; \\
& \alpha_{1}, \ldots, \alpha_{5}-\text { свободные параметры. }
\end{aligned}
$$


Также из результатов [20] (см. также [5] и [6]) следует, что асимптотика $\beta$-особых точек решений уравнений Эйлера-Пуассона имеет вид

$$
\left\{\begin{aligned}
p(t)=\widetilde{p}^{0} t^{-1}+\beta_{0} u_{0} t^{\lambda_{0}-1}+\beta^{0} u^{0} t^{\lambda^{0}-1}+\beta_{2} u_{2} t+\beta_{3} u_{3} t^{2}+\beta_{4} u_{4} t^{3} t+\cdots & \\
& +\sum_{i+j \geqslant 2} \beta_{0}^{i}\left(\beta^{0}\right)^{j} \psi_{i j} t^{i \lambda_{0}+j \lambda^{0}-1}+\cdots \\
\gamma(t)=\widetilde{\gamma}^{0} t^{-2}+\beta_{0} v_{0} t^{\lambda_{0}-2}+\beta^{0} v^{0} t^{\lambda^{0}-2}+\beta_{2} v_{2}+\beta_{3} v_{3} t+\beta_{4} v_{4} t^{2}+\cdots & \\
& +\sum_{i+j \geqslant 2} \beta_{0}^{i}\left(\beta^{0}\right)^{j} \chi_{i j} t^{i \lambda_{0}+j \lambda^{0}-2}+\cdots
\end{aligned}\right.
$$

Здесь:

$$
\widetilde{p}_{1}^{0}=\sqrt{\frac{\left(2 A_{2}-\varrho\right)\left(2 A_{3}-\varrho\right)}{B_{12} B_{31}}}, \quad \sigma,
$$

$\sigma$ - циклическая перестановка индексов $(1,2,3)$, применяемая ко всей формуле для получения оставшихся двух аналогичных формул;

$\widetilde{\gamma}^{0}$ - собственный вектор оператора $\xi \rightarrow \widetilde{p}^{0} \times \xi$ с собственным значением -2 ; $\varrho$ находится из уравнения

$$
\sum_{\sigma} r_{1}\left(A_{1}-\varrho\right) \sqrt{\left(2 A_{2}-\varrho\right)\left(2 A_{3}-\varrho\right) B_{23}}=0
$$

$\left(u_{k}, v_{k}\right), k=2,3,4,\left(u_{0}, v_{0}\right),\left(u^{0}, v^{0}\right)$ - собственные векторы оператора

$$
\mathbf{H}:(A p, \gamma) \rightarrow\left(A \widetilde{p}^{0} \times p+A p \times \widetilde{p}^{0}+\gamma \times r+A p, \widetilde{\gamma}^{0} \times p+\gamma \times \widetilde{p}^{0}+2 \gamma\right)
$$

с собственными значениями

$$
\lambda_{0}^{(0)}=\frac{1}{2} \stackrel{(+)}{-} \sqrt{\frac{1}{4}-S}, \quad S=\frac{S_{1}}{S_{2}},
$$

где

$$
\begin{gathered}
S_{1}=\frac{(2\langle A \gamma, \delta\rangle+\langle A p, p\rangle)(\langle A \gamma, \delta\rangle+\langle A p, p\rangle)}{\langle A \gamma, \gamma\rangle}-\frac{3\langle p, r\rangle^{2}}{\langle\gamma, r\rangle}-2\langle A \delta, \delta\rangle+2\langle\delta, r\rangle, \\
S_{2}=\frac{\langle p, r\rangle^{2}}{2\langle\gamma, r\rangle}-\frac{\langle A \gamma, \delta\rangle^{2}}{\langle A \gamma, \gamma\rangle}+\langle A \delta, \delta\rangle .
\end{gathered}
$$

В формуле (4.5) для простоты мы заменили $\widetilde{p}^{0}, \widetilde{\gamma}^{0}$ на $p, \gamma$, а $\delta$ удовлетворяет соотношениям $p \times \delta+2 \delta=0,\langle\delta, \gamma\rangle=2$, и, наконец, $\beta_{2}, \beta_{3}, \beta_{4}, \beta_{0}, \beta^{0}-$ свободные параметры.

Для сходимости ряда, продолжающего асимптотику (4.3), надо положить $t=e^{i\left(\bar{\lambda}_{0}-\bar{\lambda}^{0}\right) \theta}, \theta \rightarrow+\infty$.

В случае Гесса уравнение (4.4) можно решить, но удобнее сначала рассмотреть уравнение $\left\langle A \widetilde{p}^{0}, r\right\rangle=0$ :

$$
A_{1}^{2} \frac{\left(2 A_{2}-\varrho\right)\left(2 A_{3}-\varrho\right)}{B_{12} B_{31}} r_{1}^{2}=A_{2}^{2} \frac{\left(2 A_{3}-\varrho\right)\left(2 A_{1}-\varrho\right)}{B_{23} B_{12}} r_{2}^{2},
$$

корни которого равны $2 A_{3}$ и 0 и удовлетворяют (4.4). 
Пусть вначале $\varrho=2 A_{3}$. Опуская простые выкладки, приведем все же промежуточные результаты. Итак, в рассматриваемом случае в (4.5)

$$
\begin{gathered}
p=(0,0, \pm 2 i), \quad \gamma=\gamma_{1}(1, \pm i, 0), \quad \gamma_{1}=2 A_{3}\left(r_{1} \pm i r_{2}\right)^{-1} \\
\delta=\gamma_{1}^{-1}(1, \mp i, 0), \quad\langle A \gamma, \delta\rangle=A_{1}+A_{2}, \\
\langle A p, p\rangle=-4 A_{3}, \quad\langle\gamma, r\rangle=2 A_{3}, \quad\langle p, r\rangle=0, \quad\langle A \gamma, \gamma\rangle=B_{12} \gamma_{1}^{2}, \\
\langle A \delta, \delta\rangle=B_{12} \gamma_{1}^{-2}, \quad\langle\delta, r\rangle=\mathscr{R}\left(2 A_{3}\right)^{-1}, \quad S=2 A_{3} \nu^{2} \mp i \nu \sqrt{2 A_{3}} .
\end{gathered}
$$

Итак,

$$
\lambda_{0}=\mp \nu i \sqrt{2 A_{3}}, \quad \lambda^{0}=1 \pm \nu i \sqrt{2 A_{3}} .
$$

Пусть теперь $\varrho=0$. В этом случае вектор $\widetilde{p}^{0}$ равен

$$
2\left( \pm i \sqrt{\frac{A_{2} A_{3}}{-B_{12} B_{31}}}, \pm i \sqrt{\frac{A_{1} A_{3}}{-B_{12} B_{23}}},-\sqrt{\frac{A_{1} A_{2}}{B_{23} B_{31}}}\right),
$$

$\left\langle\widetilde{\gamma}^{0}, r\right\rangle=0$, формула (4.5) упрощается и принимает вид $S=6$; соответственно

$$
\lambda_{0}=-2, \quad \lambda^{0}=3 .
$$

Формальный ряд, продолжающий асимптотику (4.3), является рядом Лорана, причем заранее не ясно, как находить его коэффициенты, и, тем более, непонятно, будет ли он сходящимся. Заметим также, что в рассматриваемом случае полученный ряд для $(p, \gamma)$ не имеет неоднозначных слагаемых.

Итак, асимптотика $\alpha$-точек является однозначной. Все коэффициенты $\alpha$-точек эффективно вычисляются как функции исходных параметров тела $A_{i}, r_{i}$, $i=1,2,3$. При этом общий вид асимптотики имеет пять свободных параметров $\alpha_{i}, i=1, \ldots, 5$, что закономерно для системы (2.1) с шестью переменными.

Асимптотика $\beta$-точек также может быть однозначной, но может и иметь ветвление за счет слагаемых вида $t^{\nu}, \nu \in \mathbb{C}$. Коэффициенты при $t^{\nu}$ эффективно вычисляются не всегда, в частности когда асимптотика $\beta$-точки является частью ряда Лорана. К сожалению, именно такая ситуация имеет место в случае Гесса. Этим обстоятельством объясняется необходимость рассмотрения случая Гесса и с помощью уравнения Риккати для $\varphi$, и с помощью линейной системы уравнений относительно $\theta_{1}, \theta_{2}$.

Координаты особых точек наиболее просто находятся для функции $\theta$, а асимптотика соответствующих особых точек - для функции $\varphi$.

Определяя соответствие особых точек для различных представлений решения задачи, мы можем получить необходимые нам свойства решения для каждого из представлений.

Поскольку функция $\mathscr{F}$ является эллиптической, то можно считать, что уравнение (3.7) для $\varphi$ и система (3.8) для $\theta$ заданы на $\mathbb{R}$-двумерном торе $T^{2}$, являющемся голоморфным компактным многообразием. Однако в правой части $(3.7),(3.8)$ вместе с $\mathscr{F}$ присутствует и $\sqrt{\mathscr{F}}$, что требует рассмотрения минимального накрытия, для которого правые части уравнений $(3.7),(3.8)$ становятся однозначными. 
Функция $\mathscr{F}$ имеет полюс второго порядка, значит, в окрестности точки $\mathscr{F}=\infty$ функция $\sqrt{\mathscr{F}}$ однозначна. Точки $\mathscr{F}=0$ не могут принадлежать вещественной прямой, так как при $\mathscr{F}=0$ имеем $A_{3}^{2}(\dot{\mathscr{F}})^{2}=-\mathscr{M}^{2} \mathscr{R}<0$, если $\mathscr{M} \neq 0$.

Как было отмечено в замечании 1 , случай $\mathscr{M}=0$ явно интегрируется, поэтому в данном случае не интересен для рассмотрения.

Точки, в которых $\mathscr{F}=0$, в силу вещественности $\mathscr{F}$ имеют сопряженные координаты, значит, они отличны друг от друга и не являются кратными. Риманова поверхность функции $\sqrt{\mathscr{F}}$ согласно формуле Римана-Гурвица является поверхностью $S_{2} 2$-го рода (см. [18]; это можно видеть и из геометрии разветвленного накрытия).

ЗАмЕЧАниЕ 4. В силу линейности уравнений для $\theta$ соответствующий им поток можно профакторизовать по однородному растяжению, после чего мы получаем голоморфное слоение с особенностями на компактном голоморфном многообразии $S_{2} \times \mathbb{C} P^{1}$. При этом очевидно соответствие между $\left(\theta_{1}: \theta_{2}\right) \in \mathbb{C} P^{1}$ и $\varphi=\theta_{1} / \theta_{2}$.

Для вещественных $\varphi$ и $\mathscr{F}$ мы получаем два инвариантных тора с условиями $\sqrt{\mathscr{F}}>0$ и $\sqrt{\mathscr{F}}<0$.

Функция $\mathscr{F}$ является в общем случае вещественной эллиптической функцией, поэтому ее периоды имеют вид $\omega_{1}, i \omega_{2}, \omega_{i} \in \mathbb{R}$. Кроме того, точки, в которых $\mathscr{F}=0$ или $\mathscr{F}=\infty$, являются сопряженными, поэтому далее, получая асимптотики функций $\mathscr{F}, \varphi, \theta$ в этих точках, мы будем указывать только одну из них. Из дифференциального уравнения (2.5) стандартным образом получаем асимптотику особых точек $\mathscr{F}$.

Предложение 2. Асимптотика функиий $\mathscr{F}, \sqrt{\mathscr{F}}, \mathscr{F}^{-1}$ в точке $\mathscr{F}=\infty$ имеет вид

$$
\begin{aligned}
\mathscr{F} & =-\frac{2 A_{3}}{t^{2}}+\frac{2}{3} \mathscr{H}+\left(\frac{\sigma}{10}-\frac{2 \mathscr{H}^{2}}{15 A_{3}}\right) t^{2}+\cdots, \quad \sigma=\frac{\mathscr{H}^{2}-\mathscr{T} \mathscr{R}}{A_{3}}, \\
\mathscr{F}^{-1} & =-\frac{t^{2}}{2 A_{3}}-\frac{\mathscr{H}}{6 A_{3}^{2}} t^{4}-\frac{1}{5 A_{3}^{2}}\left(\frac{\sigma}{8}+\frac{\mathscr{H}^{2}}{9 A_{3}}\right) t^{6}+\cdots, \\
\sqrt{\mathscr{F}} & =\frac{\lambda}{t}+\frac{\mathscr{H}}{3 \lambda} t+\left(\frac{\sigma}{20 \lambda}-\frac{\mathscr{H}^{2}}{15 A_{3} \lambda}-\frac{\mathscr{H}^{2}}{18 \lambda^{3}}\right) t^{3}+\cdots, \quad \lambda=i \sqrt{2 A_{3}} .
\end{aligned}
$$

Асимптотика функиий $\mathscr{F}, \sqrt{\mathscr{F}}, \mathscr{F}^{-1}$ в одной из сопряженных точек $\mathscr{F}=0$ имеет вид

$$
\begin{aligned}
\mathscr{F} & =\mu t-\frac{\sigma}{2} t^{2}+\frac{2 \mathscr{H} \mu}{3 A_{3}} t^{3}+\cdots, \quad \mu=4 i \kappa=\frac{i \mathscr{M} \sqrt{\mathscr{R}}}{A_{3}}, \\
\mathscr{F}^{-1} & =\frac{1}{\mu t}+\frac{\sigma}{2 \mu^{2}}+\left(\frac{\sigma^{2}}{4 \mu^{3}}-\frac{2 \mathscr{H}}{3 A_{3} \mu}\right) t+\cdots, \\
\sqrt{\mathscr{F}} & =\sqrt{\mu} t^{1 / 2}-\frac{\sigma}{4 \sqrt{\mu}} t^{3 / 2}+\left(\frac{\sqrt{\mu} \mathscr{H}}{3 A_{3}}-\frac{\sigma^{2}}{32 \mu^{3 / 2}}\right) t^{5 / 2}+\cdots
\end{aligned}
$$


Как уже было отмечено, асимптотика функций $\mathscr{F}, \sqrt{\mathscr{F}}, \mathscr{F}^{-1}$ находится из (2.5).

Из дифференциального уравнения (3.7) находим асимптотику функции $\varphi$.

ПРЕДЛОЖЕНИЕ 3. Функиия $\varphi$ в окрестности точки $\mathscr{F}=0, \mathscr{F}=4 i \kappa t+\cdots$ nри $t \rightarrow 0$ имеет с точностью до комплексного сопряжения следующие асимптотики:

$$
\varphi=-i+c t^{1 / 2}+\frac{i c^{2}}{2} t-\left(\frac{i c \sigma}{16 \kappa}+\frac{c^{3}}{4}+2 \nu i \sqrt{\kappa i}\right) t^{3 / 2}+\cdots,
$$

где с-свободный параметр, либо

$$
\varphi=i+\nu i \sqrt{\kappa i} t^{3 / 2}+\cdots .
$$

ДокАЗАТЕЛЬСтво. Итак, согласно условию $\mathscr{F}=4 i \kappa t+\cdots$ при $t \rightarrow 0$. Предположим, что при этом $1+\varphi^{2}$ (см. (3.7)) не стремится к нулю, т.е. $\varphi$ не стремится к $\pm i$. В этом случае $\nu \sqrt{\mathscr{F}} \varphi$ мало по сравнению с $-\frac{\kappa}{\mathscr{F}}\left(\varphi^{2}+1\right)$, поэтому

$$
\begin{aligned}
\dot{\varphi} & =\frac{i}{4 t}\left(\varphi^{2}+1\right)+\cdots, \quad t \rightarrow 0 \\
& \Longrightarrow \quad \varphi=\operatorname{tg}\left(\frac{i}{4} \ln (c t)\right)+\cdots=-i \frac{(c t)^{-1 / 4}-(c t)^{1 / 4}}{(c t)^{-1 / 4}+(c t)^{1 / 4}} \rightarrow-i, \quad t \rightarrow 0 .
\end{aligned}
$$

Мы получаем противоречие, значит, в сопряженных точках $\varphi \rightarrow \mp i$ при $\mathscr{F}=0$.

Пусть $\mathscr{F}=4 i \kappa t+\cdots$ при $t \rightarrow 0$ и $\varphi \rightarrow-i$.

Подставим $\varphi=-i+\varphi_{1}$ в уравнение $(3.7)$ :

$$
\dot{\varphi}_{1}=\nu \sqrt{\mu t} \varphi_{1}+\frac{1}{2 t} \varphi_{1}+\cdots, \quad t \rightarrow 0, \quad \varphi_{1} \rightarrow 0,
$$

и получим приближенное решение $\varphi_{1}$, а значит, и $\varphi$ :

$$
\varphi=-i+c t^{1 / 2}+\cdots, \quad t \rightarrow 0
$$

$c$ - свободный параметр. Последующие итерации дают искомый результат.

Пусть $\mathscr{F}=4 i \kappa t+\cdots$ при $t \rightarrow 0$ и $\varphi \rightarrow i$. В этом случае мы не можем найти однопараметрическое семейство асимптотик, но одна искомая асимптотика все же имеется:

$$
\varphi=i+\nu i \sqrt{\kappa i} t^{3 / 2}+\cdots .
$$

Рассмотрим возмущение этого решения. Полагая

$$
\varphi=i+\nu i \sqrt{\kappa i} t^{3 / 2}+\varepsilon \Phi(t)
$$

и пренебрегая слагаемыми с $\varepsilon$ порядка выше 1 , имеем $\Phi(t)=-c t^{-1 / 2}+\cdots$. Это означает, что при малом возмущении рассматриваемого решения $\varphi$ не стремится к $i$ при $t \rightarrow 0$.

Как уже было показано, $\varphi \rightarrow-i$, если $\varphi$ не стремится к $i$, значит, обе приведенные асимптотики исчерпывают все возможности для решения $\varphi$ при условии $\mathscr{F}=4 i \kappa t+\cdots, t \rightarrow 0$. 
ПРеДЛОЖенИЕ 4. Асимптотика функиии ч в окрестности точки $\mathscr{F}=\infty$ имеет вид

$$
\varphi=c t^{\lambda \nu}+\cdots+\frac{\kappa t^{3}}{2 A_{3}(3-\lambda \nu)}+\cdots, \quad \nu=-\frac{\sqrt{2 B_{23} B_{31}}}{\sqrt{A_{1} A_{2} A_{3}}}, \quad \lambda=i \sqrt{2 A_{3}},
$$

либо

$$
\varphi=-\frac{2 A_{3}(3+\lambda \nu)}{\kappa t^{3}}-\frac{2 H(3+\nu \lambda)\left(A_{3} \nu-(3+\nu \lambda) \lambda\right)}{3 \kappa \lambda(5+\lambda \nu) t} \cdots .
$$

ДокАЗАТЕЛЬСтво. Предположим, что $\mathscr{F} \rightarrow \infty$ при $t \rightarrow 0$; тогда либо

$$
\dot{\varphi}=\nu \sqrt{\mathscr{F}} \varphi+\cdots \quad \Longrightarrow \quad \ln \varphi=\nu \lambda \ln t+\cdots \quad \Longrightarrow \quad \varphi=c t^{\lambda \nu}+\cdots
$$

и далее применяем стандартные итерации, либо

$$
\dot{\varphi}=-\frac{\kappa \varphi^{2}}{\mathscr{F}}+\cdots=\frac{\kappa \varphi^{2} t^{2}}{2 A_{3}}+\cdots,
$$

и получаем

$$
\varphi=-\frac{2 A_{3}(3+\lambda \nu)}{\kappa t^{3}}+\cdots
$$

Найдем теперь асимптотики функций $\theta_{1}, \theta_{2}$ в окрестностях точек $\mathscr{F}=0$, $\mathscr{F}=\infty$.

Функции $\theta_{1}, \theta_{2}$ удовлетворяют (см. (3.8)) системе дифференциальных уравнений

$$
\left\{\begin{array}{l}
\dot{\theta}_{1}=\frac{\nu}{2} \sqrt{\mathscr{F}} \theta_{1}-\frac{\kappa}{\mathscr{F}} \theta_{2}, \\
\dot{\theta}_{2}=\frac{\kappa}{\mathscr{F}} \theta_{1}-\frac{\nu}{2} \sqrt{\mathscr{F}} \theta_{2} .
\end{array}\right.
$$

Поскольку $\varphi=\theta_{1} / \theta_{2}$, то из второго уравнения этой системы следует

$$
\left(\ln \theta_{2}\right)^{\cdot}=\frac{\kappa}{\mathscr{F}} \varphi-\frac{\nu}{2} \sqrt{\mathscr{F}} .
$$

Необходимые асимптотики $\varphi, \mathscr{F}, \sqrt{\mathscr{F}}$ уже известны, поэтому несложно получить и асимптотики $\theta_{i}$.

ПРЕДЛОЖЕНИЕ 5. В окрестности точки $\mathscr{F}=0$ функции $\theta_{i}$ с точностъю до сопря⿻ения имеют асимптотику

$$
\left\{\begin{array}{l}
\theta_{1}=-i c_{1} t^{-1 / 4}+i c_{2} t^{1 / 4}+\cdots \\
\theta_{2}=c_{1} t^{-1 / 4}+c_{2} t^{1 / 4}+\cdots
\end{array}\right.
$$

где $c_{1}, c_{2}$ - свободные параметры.

ЗАмечАниЕ 5. Функция $\varphi$ в окрестности точки $\mathscr{F}=0$ имеет две различные асимптотики. Обе они соответствуют асимптотике функций $\theta_{i}$ из доказанного предложения: асимптотика (4.6) соответствует случаю $c_{1} \neq 0$, асимптотика (4.7) - случаю $c_{1}=0$. 
ПРеДЛОЖенИЕ 6. В окрестности точки $\mathscr{F}=\infty$ функиии $\theta_{i}$ имеют асимnmотику

$$
\left\{\begin{array}{l}
\theta_{1}=c_{1} t^{\nu \lambda / 2}+c_{1} \frac{\nu \mathscr{H}}{12 \lambda} t^{2+\nu \lambda / 2}+c_{2} \frac{\kappa}{2 A_{3}(3-\nu \lambda)} t^{3-\nu \lambda / 2}+\cdots, \\
\theta_{2}=c_{2} t^{-\nu \lambda / 2}-c_{2} \frac{\nu \mathscr{H}}{12 \lambda} t^{2-\nu \lambda / 2}-c_{1} \frac{\kappa}{2 A_{3}(3+\nu \lambda)} t^{3+\nu \lambda / 2}+\cdots,
\end{array}\right.
$$

где $c_{1}, c_{2}$ - свободнъе параметры.

ЗАмечАниЕ 6 . Функция $\varphi$ в окрестности точки $\mathscr{F}=\infty$ имеет две различные асимптотики. Обе они соответствуют асимптотике функций $\theta_{i}$ из доказанного предложения: асимптотика (4.8) соответствует случаю $c_{2} \neq 0$, асимптотика (4.9) - случаю $c_{2}=0$.

Определим теперь соответствие особых точек решений $(p, \gamma), \theta, \varphi$.

ОПРЕДЕЛЕНИЕ 1 . Будем называть $\beta(\varrho)$-особъми точками $\beta$-особые точки, соответствующие корню $\varrho$ уравнения (4.4).

ПРеДЛОЖениЕ 7. Решения $(p, \gamma)$ имеют особъе точки типа $\beta\left(2 A_{3}\right)$, если и только если $\mathscr{F} \rightarrow \infty$.

ДокАЗАТЕЛЬство. Необходимость. Пусть $\mathscr{F} \nrightarrow \infty$. Тогда $p \rightarrow \infty$ только при условии $\varphi \rightarrow \pm i$, но тогда $\left(p_{1}: p_{2}: p_{3}\right) \rightarrow\left( \pm k_{1}: \pm i k_{2}: 2 k_{3}\right)$, а значит, рассматриваемые особые точки не являются $\beta\left(2 A_{3}\right)$-точками.

Достаточность. Пусть $\mathscr{F} \rightarrow \infty$. Согласно $(2.4)(p, \gamma) \rightarrow \infty$ и при этом $\left(p_{1}: p_{2}: p_{3}\right)=\left(k_{1}: k_{2}:\left(1-\varphi^{2}\right) / \varphi\right)$. Из асимптотики $\varphi$ в точке $\mathscr{F}=\infty$ следует, что если входить в особую точку по спирали $t=e^{i\left(\lambda_{0}-\lambda^{0}\right) \theta}, \theta \rightarrow+\infty$, то $\varphi \rightarrow 0$ или $\varphi \rightarrow \infty$ в зависимости от знака направления вращения спирали, значит, $\left(1-\varphi^{2}\right) / \varphi \rightarrow \infty$ и $\left(p_{1}: p_{2}: p_{3}\right) \rightarrow(0: 0: 1)$. Ясно, что рассматриваемая особая точка не является ни $\alpha$-точкой, ни $\beta(0)$-точкой, а по теореме о полной классификации особых точек (см. [20]) может быть только $\beta\left(2 A_{3}\right)$-точкой.

ЗАмечАниЕ 7. Предложение можно было бы доказать и не ссылаясь на классификационную теорему, но тогда необходимо по формулам (2.4) более точно определить асимптотику $(p, \gamma)$ при $\mathscr{F} \rightarrow \infty$.

Дополнительным обстоятельством, подтверждающим предложение, является наличие ветвления одного и того же вида $\left(t^{\nu \sqrt{2 A_{3}}}\right)$ и для $\beta\left(2 A_{3}\right)$-точки, и для $\varphi$ при $\mathscr{F} \rightarrow \infty$.

ПрЕДЛОЖЕНИЕ 8. Пусть $\varphi \rightarrow \pm i$. Тогда:

1) если $\mathscr{F} \rightarrow \mathscr{F}_{0} \neq 0, \infty$, то решение $(p, \gamma)$ имеет $\alpha$-особую точку;

2) если $\mathscr{F}=4 i \kappa t+\cdots$ nри $t \rightarrow 0, \varphi \rightarrow-i u$

2.1) $c \neq 0$ ( $(p, \gamma)$ не имеет особых точек,

2.2) $c=0$, то решение $(p, \gamma)$ имеет $\alpha$-особую точку;

3) если $\mathscr{F}=4 i \kappa t+\cdots$ при $t \rightarrow 0, \varphi \rightarrow i$, то решение $(p, \gamma)$ имеет $\beta(0)$ особую точку.

Для доказательства достаточно подставить асимптотики $\varphi$ в (2.4). 


\section{§5. Параметрическое отображение последования для вещественных решений случая Гесса}

В этом параграфе доказываются формулы (2.13)-(2.19) теоремы 1.

ОПРЕДЕЛЕНИЕ 2. Рассмотрим слоение, заданное на компактном голоморфном многообразии $T^{2} \times \mathbb{C} P^{1}$ факторизацией потока уравнений Эйлера-Пуассона в случае Гесса. Здесь $T^{2}$ - тор, задаваемый двоякопериодической функцией $\mathscr{F}$, а $\mathbb{C} P^{1}$ - область значений фунции $\varphi$. Пусть $\gamma:[0,1] \rightarrow T^{2}-$ путь на $T^{2}$, соединяющий две особые точки (возможно, совпадающие) $\gamma(0)$ и $\gamma(1)$, в которых $\mathscr{F}\left(t_{*}\right)=\infty$ (или $\left.\mathscr{F}\left(t_{0}\right)=0\right)$. Путь $\gamma$ в силу имеющегося слоения поднимается в расслоение $T^{2} \times \mathbb{C} P^{1} \rightarrow T^{2}$ и задает отображение $\Phi_{\gamma}: \mathbb{C} P^{1} \rightarrow \mathbb{C} P^{1}$ параметров, определяющих асимптотику фукнции $\varphi(t)$ в особых точках. Отображение $\Phi_{\gamma}$ будем называть параметрическим отображением последования.

ЗАМЕЧАНИЕ 8. В отличие от классического определения отображения последования данное определение относится к предельному отображению окрестностей особых точек. Записанное в координатах, это отображение является вектор-функцией из пространства свободных параметров одной особой точки в соответствующее пространство другой особой точки.

Важность такого понятия заключается в том, что одно значение параметра асимптотики определяет целое решение $\varphi(t)$ при том, что асимптотики решений $\varphi(t)$ в особых точках могут быть найдены.

Рассмотрим параметрическое отображение последования за период $\tau_{1}$ для особых точек, находящихся над вещественной прямой, функции $\mathscr{F}$ вдоль вещественной оси времени. Для функции $\varphi$ это будет дробно-линейное отображение; оно имеет вид

$$
\Phi_{\tau_{1}}: c \rightarrow \frac{a_{11} c+a_{12}}{a_{21} c+a_{22}}, \quad \operatorname{det}\left(\begin{array}{ll}
a_{11} & a_{12} \\
a_{21} & a_{22}
\end{array}\right)=1 .
$$

Для особых точек, находящихся под вещественной прямой, параметрическое отображение последования за период $\tau_{1}$ будет сопряженным к отображению (5.1). Но это же отображение совпадает с отображением (5.1), но на двойственном листе $\sqrt{\mathscr{F}}$, поскольку функция $\mathscr{F}$ периодична с периодом $i \tau_{2}$, а функция $\sqrt{\mathscr{F}}-$ с периодом $2 i \tau_{2}$.

Чтобы различать отображения $\Phi_{\tau_{1}}$, добавляем в них к нижним индексам плюс и минус.

Пусть $\Phi_{\tau_{1}+}$ есть отображение (5.1); тогда

$$
\Phi_{\tau_{1}-}: c \rightarrow-\frac{a_{22} c-a_{21}}{a_{12} c-a_{11}} .
$$

Учитывая, что оператор $\Phi_{\tau_{1}+} \bar{\Phi}_{\tau_{1}-}^{-1}$ задается обходом вокруг особой точки с ветвлением, т.е. представляет собой умножение на $\delta^{2}=\exp \left(2 \pi \nu \sqrt{2 A_{3}}\right)$, получаем соотношения

$$
a_{11}=\delta^{2} \bar{a}_{22}, \quad a_{21}=-\delta^{2} \bar{a}_{12}, \quad \delta^{2}=\exp \left(2 \pi \nu \sqrt{2 A_{3}}\right) .
$$


Рассмотрим теперь параметрическое отображение последования за период $\tau_{2}$ функции $\mathscr{F}$ вдоль мнимой оси времени от особой точки под вещественной осью к особой точке над осью. Для функции $\varphi$ это будет, как и $\Phi_{\tau_{1} \pm}$, дробно-линейное отображение; оно имеет вид

$$
\Phi_{\tau_{2}+}: c \rightarrow \frac{b_{11} c+b_{12}}{b_{21} c+b_{22}}, \quad \operatorname{det}\left(\begin{array}{ll}
b_{11} & b_{12} \\
b_{21} & b_{22}
\end{array}\right)=1 .
$$

Вещественные решения, т.е. решения, принимающие вещественные значения на вещественной оси, выделяются условием: $\Phi_{\tau_{2}+}(\bar{c})=c$, или

$$
b_{21} c \bar{c}+b_{22} c-b_{11} \bar{c}-b_{12}=0 .
$$

Известно, что движения сферы стереографической проекции порождают на комплексной плоскости дробно-линейное отображение. Следовательно, $\Phi_{\tau_{1}+}$ порождает слоение комплексной плоскости на инвариантные окружности, одна из которых соответствует вещественному решению.

В таком случае уравнение (5.4) должно задавать окружность, а именно ту из инвариантных окружностей (5.1) отображения $\Phi_{\tau_{1}+}$, которой соответствует вещественное решение. Коэффициенты этого уравнения должны удовлетворять следующим условиям:

$$
\left|b_{11}\right|=\left|b_{22}\right|, \quad \frac{b_{22}}{b_{21}}=-\frac{\bar{b}_{11}}{\bar{b}_{21}}, \quad \frac{b_{12}}{b_{21}} \in \mathbb{R},
$$

и в этом случае инвариантная окружность имеет центр в точке $b_{11} / b_{21}$ и радиус

$$
R=\sqrt{\frac{b_{12}}{b_{21}}+\left|\frac{b_{11}}{b_{21}}\right|^{2}}
$$

ЗАмЕчАниЕ 9. Условия (5.5) можно получить также следующим образом:

$$
\Phi_{\tau_{2}+}(\bar{c})=c \quad \Longrightarrow \quad \Phi_{\tau_{2}+} \bar{\Phi}_{\tau_{2}+}(c)=\bar{c} \quad \Longrightarrow \quad \Phi_{\tau_{2}+} \bar{\Phi}_{\tau_{2}+}=E,
$$

и далее приходим к системе

$$
\left\{\begin{array}{l}
\left|b_{11}\right|+b_{12} \bar{b}_{21}=1 \\
\left|b_{22}\right|+\bar{b}_{12} b_{21}=1 \\
b_{11} \bar{b}_{12}+b_{12} \bar{b}_{21}=0 \\
b_{11} \bar{b}_{21}+b_{21} \bar{b}_{22}=0 .
\end{array}\right.
$$

Сравнивая полученное уравнение окружности (5.4) с уравнением

$$
\left(z-z_{0}\right)\left(\bar{z}-\bar{z}_{0}\right)=z \bar{z}-\bar{z}_{0} z-z_{0} \bar{z}+z_{0} \bar{z}_{0}=R^{2},
$$

получаем

$$
b_{11}=z_{0} b_{21}, \quad b_{22}=-\bar{z}_{0} b_{21}, \quad b_{12}=\left(R^{2}-z_{0} \bar{z}_{0}\right) b_{21} .
$$


Наконец, поскольку определитель матрицы $\Phi_{\tau_{2}}+$ равен 1 , то

$$
-z_{0} \bar{z}_{0} b_{21}^{2}-\left(R^{2}-z_{0} \bar{z}_{0}\right) b_{21}^{2}=-R^{2} b_{21}^{2}=1 \quad \Longrightarrow \quad b_{21}= \pm \frac{i}{R},
$$

и окончательно получаем

$$
b_{21}= \pm \frac{i}{R}, \quad b_{11}= \pm \frac{i z_{0}}{R}, \quad b_{22}=\bar{b}_{11}, \quad b_{12}=\left(R^{2}-z_{0} \bar{z}_{0}\right) b_{21} .
$$

Из матричного равенства

$$
\Phi_{\tau_{2}+} \bar{\Phi}_{\tau_{1}+}=\Phi_{\tau_{1}+} \Phi_{\tau_{2}+}
$$

получаем

$$
a_{11}, a_{22} \in \mathbb{R}, \quad b_{21}=-\delta^{2} b_{12}, \quad z_{0} \bar{z}_{0}-R^{2}=\delta^{-2},
$$

а также

$$
a_{11}-a_{22}=2 \operatorname{Re}\left(a_{21} \frac{b_{11}}{b_{21}}\right) .
$$

Последнее условие получается так же, как условие совпадения инвариантных окружностей отображений $\Phi_{\tau_{1}+}$ и $\Phi_{\tau_{2}+}$.

Отметим также, что отображение последования $\Phi_{\tau_{1}+}$ имеет неподвижные точки

$$
c_{ \pm}=\frac{a_{11}-a_{22} \pm \sqrt{\left(a_{11}+a_{22}\right)^{2}-4}}{2 a_{21}},
$$

являющиеся корнями квадратного уравнения

$$
a_{21} c^{2}+\left(a_{22}-a_{11}\right) c-a_{12}=0
$$

и определяющие асимптотику особых точек периодических решений.

Если выполнено условие

$$
\left|a_{11}+a_{22}\right|<2
$$

то отображение последования $\Phi_{\tau_{1}+}(c)$ имеет собственные значения

$$
\lambda_{ \pm}=\frac{a_{11}+a_{22} \pm \sqrt{\left(a_{11}+a_{22}\right)^{2}-4}}{2}=\exp ( \pm i \alpha), \quad \alpha \in \mathbb{R},
$$

т.е. образы степеней отображения последования некоторой начальной точки образуют квазипериодическую последовательность на инвариантной окружности, что означает, что все решения $\varphi(t)$ в этом случае квазипериодичны.

Естественно, что при этом центры инвариантных окружностей совпадают с неподвижными точками (5.10).

Если выполнено условие

$$
\left|a_{11}+a_{22}\right|>2
$$

то собственные значения $\lambda_{ \pm}$отображения последования $\Phi_{\tau_{1}+}(c)$ вещественны.

При бесконечном итерировании отображения $\Phi_{\tau_{1}+}(c)$ образ $\Phi_{\tau_{1}+}^{n}(c), n \in \mathbf{Z}^{+}$, точки $c$ будет стремиться к $c_{+}$. Соответственно, при итерировании $\Phi_{\tau_{1}+}^{-1}$ ситуация аналогична: $\Phi_{\tau_{1}+}^{-n} c \rightarrow c_{-}$. Таким образом, предельными периодическими 
решениями функции $\varphi$ будут периодическая функция $\varphi_{+}$с постоянным свободным параметром асимптотики $c_{+}$при $t \rightarrow+\infty$ и периодическая функция $\varphi_{-}$ с постоянным свободным параметром асимптотики $c_{-}$при $t \rightarrow-\infty$.

Наконец, исходя из вышеизложенного, $\Phi_{\tau_{2}-}$ находим аналогично $\Phi_{\tau_{1}-}$ :

$$
\Phi_{\tau_{2}-}: c \rightarrow-\frac{b_{22} c-b_{21}}{b_{12} c-b_{11}} .
$$

\section{$\S 6$. Однозначные решения}

\section{уравнений Эйлера-Пуассона в случае Гесса}

ТеОРема 2. Все однозначные решения уравнений Эйлера-Пуассона (2.1) в случае Гесса имеют вид

$$
\left\{\begin{array}{l}
p_{1}(t) \equiv p_{2}(t) \equiv \gamma_{3}(t) \equiv 0, \\
p_{3}(t)=\sqrt{\frac{2 \mathscr{F}}{A_{3}}}, \\
\gamma_{1}=\frac{1}{\mathscr{R}}\left(r_{1}(\mathscr{H}-\mathscr{F})+A_{3} r_{2} \dot{p}_{3}\right), \\
\gamma_{2}=\frac{1}{\mathscr{R}}\left(r_{2}(\mathscr{H}-\mathscr{F})-A_{3} r_{1} \dot{p}_{3}\right),
\end{array}\right.
$$

где $\mathscr{F}$ есть эллиптическая функиия, задаваемая уравнением (см. (2.5))

$$
A_{3}^{2}(\dot{\mathscr{F}})^{2}=2 A_{3} \mathscr{T} \mathscr{R} \mathscr{F}-2 A_{3} \mathscr{F}(\mathscr{F}-\mathscr{H})^{2},
$$

$\mathscr{H}, \mathscr{T}$ - значения интеграла энергии (3.2) и тривиального интеграла (3.4) соответственно (при этом интеграл $\mathscr{M}$ момента (3.3) решения равен нулю), или находятся из решения линейной системь (см. (3.8))

$$
\left\{\begin{array}{l}
\dot{\theta}_{1}=\frac{\nu}{2} \sqrt{\mathscr{F}_{0}} \theta_{1}-\frac{\kappa}{\mathscr{F}_{0}} \theta_{2} \\
\dot{\theta}_{2}=\frac{\kappa}{\mathscr{F}_{0}} \theta_{1}-\frac{\nu}{2} \sqrt{\mathscr{F}_{0}} \theta_{2}
\end{array}\right.
$$

$\mathscr{F}_{0}$ - корень полинома (см. (2.5))

$$
\mathscr{P}_{3}(\mathscr{F})=2 A_{3} \mathscr{T} \mathscr{R} \mathscr{F}-2 A_{3} \mathscr{F}(\mathscr{F}-\mathscr{H})^{2}-\mathscr{M}^{2} \mathscr{R}
$$

в этом случае

$$
\left\{\begin{array}{l}
p_{i}=k_{i} \sqrt{\mathscr{F}_{0}} \frac{\varphi}{1+\varphi^{2}}, \quad i=1,2, \\
p_{3}=k_{3} \sqrt{\mathscr{F}_{0}} \frac{1-\varphi^{2}}{1+\varphi^{2}}, \\
\gamma_{1}=\frac{1}{\mathscr{R}}\left(r_{1}\left(\mathscr{H}-\mathscr{F}_{0}\right)+r_{2}\left(A_{3} \dot{p}_{3}-B_{12} p_{1} p_{2}\right)\right), \\
\gamma_{2}=\frac{1}{\mathscr{R}}\left(r_{2}\left(\mathscr{H}-\mathscr{F}_{0}\right)-r_{1}\left(A_{3} \dot{p}_{3}-B_{12} p_{1} p_{2}\right)\right), \\
\gamma_{3}=\frac{1}{r_{2}}\left(B_{23} p_{2} p_{3}-A_{1} \dot{p}_{1}\right), \\
k_{2}=-\frac{A_{1} r_{1}}{\theta_{2} r_{2}} k_{1}, \quad k_{1}=2 \sqrt{\frac{2 B_{23}}{-B_{12} A_{1}}}, \quad k_{3}=\sqrt{\frac{2}{A_{3}}} .
\end{array}\right.
$$


Все решения, отличные от приведенных выше, имеют $\beta\left(2 A_{3}\right)$-особые точки ветвления с асимптотикой, в которой имеются с точностъю до коэфбициента слагаемые $t^{n_{1}+n_{2} \nu i \sqrt{2 A_{3}}}, n_{1}, n_{2} \in \mathbb{N}$.

ДокАЗАтельство. Рассмотрим задачу о движении тяжелого твердого тела (2.1) в случае Гесса и ее представление (3.7) с помощью уравнения Риккати. Будем считать, что значения первых интегралов $\mathscr{H}, \mathscr{M}, \mathscr{T}$ фиксированы.

Функция $\mathscr{F}$ может быть постоянной, только если она тождественно равна корню полинома правой части (2.5). Тогда мы получаем линейную систему дифференциальных уравнений с постоянными коэффициентами для нахождения $\theta_{i}$ и далее очевидным образом получаем однозначное решение задачи Гесса.

Рассмотрим теперь случай, когда функция $\mathscr{F}$ не является константой. Начнем со случая общего положения, когда $\mathscr{F}$ - двоякопериодическая функция. Так как функция $\mathscr{F}$ не является константой, то в некоторых точках она стремится в бесконечность. Как следует из предложения 6 , особые точки решения $\varphi$ при $\mathscr{F} \rightarrow \infty$ соответствуют $\beta\left(2 A_{3}\right)$-особым точкам функций $(p, \gamma)$. Почти все эти решения имеют ветвление $t^{n_{1}+n_{2} \nu i \sqrt{2 A_{3}}}$. Исключение составляет решение $(p, \gamma)$, в асимптотике которого $\beta^{0}=\beta_{0}=0$, а оставшиеся параметры $\beta_{2}, \beta_{3}, \beta_{4}$ однозначно определяются значениями первых интегралов $\mathscr{H}, \mathscr{M}, \mathscr{T}$. Это означает, что такое решение является двоякопериодическим. Но тогда сумма вычетов функции $p(t)$ должна равняться нулю (см. [18]). Для $\beta\left(2 A_{3}\right)$-особых точек это условие выполнено, а для $\alpha$ - и $\beta(0)$-точек - нет. Следовательно, в однозначном решении их не должно быть совсем. Но тогда функции $p_{1}(t), p_{2}(t)$ вообще не имеют особых точек и, следовательно, являются константами.

С помощью представления (2.4) выражаем $\varphi$ через $\mathscr{F}$ :

$$
\varphi=\frac{k_{i} \sqrt{\mathscr{F}}+\sqrt{k_{i}^{2} \mathscr{F}-4 p_{i 0}^{2}}}{2 p_{i 0}}, \quad i=1,2 .
$$

Если $p_{10}, p_{20} \neq 0$, то подставляем полученное представление в (3.7) и убеждаемся в том, что ни при каких значениях первых интегралов тождества нет.

Если $p_{10} \equiv p_{20} \equiv 0$, то из (2.4) следует $\varphi \equiv 0$, и мы получаем искомое решение, которое, как легко видеть, имеет место лишь при $\mathscr{M}=0$.

Случай, когда полином в правой части (2.5) имеет кратные корни, является предельным для уже рассмотренного случая общего положения, когда кратных корней нет. Поэтому то, что однозначное решение возможно только при условии $\mathscr{M}=0$, остается верным и для случая кратных корней и получается в результате предельного перехода.

Итак, рассмотрены все возможные случаи, значит, найдены все однозначные решения уравнений Эйлера-Пуассона в случае Гесса.

ЗАмЕчАниЕ 10. Все решения, полученные в теореме 1, хорошо известны и приведены в конечном виде в [22].

ЗАмечАниЕ 11. Доказательство теоремы 2 об однозначных решениях практически без изменений можно повторить при поиске конечнозначных решений 
(см. [21]), поскольку все асимптотики решений в случае Гесса либо однозначны, либо бесконечнозначны.

Таким образом, верно следующее утверждение.

ПрЕДЛОЖЕНИЕ 9. Все конечнозначные решения задачи Гесса являются однозначными.

ЗАмечАние 12. Как показал А. М. Ляпунов (см. [23] и [24]), все решения задачи о движении тяжелого твердого тела являются однозначными только в случаях Эйлера, Лагранжа и Ковалевской. Для других случаев движения твердого тела эта задача не ставилась, но, как видно из предложенного рассмотрения случая Гесса, такая задача вполне содержательна.

\section{§ 7. Почти периодические, асимптотически периодические и периодические решения задачи Гесса}

Теорема 3. Система дифференциальных уравнений (2.1) в случае Гесса (2.3) имеет два вещественных периодических решения, а все остальные асимптотически приближаются к одному из них на тех инвариантных торах, на которых в условиях теоремы 1

$$
\left|x_{2}^{3 / 2}\right| \geqslant 2 \kappa .
$$

В этом случае для периодических решений $\varphi_{\text {major }}, \varphi_{\text {minor }}$ имеют место оценки

$$
\begin{aligned}
& \frac{\nu x_{2}^{3 / 2}+\sqrt{\nu^{2} x_{2}^{3}-4 \kappa^{2}}}{2 \kappa} \leqslant \varphi_{\text {major }} \leqslant \frac{\nu x_{3}^{3 / 2}+\sqrt{\nu^{2} x_{3}^{3}-4 \kappa^{2}}}{2 \kappa}, \\
& \frac{\nu x_{3}^{3 / 2}-\sqrt{\nu^{2} x_{3}^{3}-4 \kappa^{2}}}{2 \kappa} \leqslant \varphi_{\text {minor }} \leqslant \frac{\nu x_{2}^{3 / 2}-\sqrt{\nu^{2} x_{2}^{3}-4 \kappa^{2}}}{2 \kappa} .
\end{aligned}
$$

Между решениями $\varphi_{\text {major }}$ и $\varphi_{\text {minor }}$ существует зависимость

$$
\varphi_{\text {minor }}(t)=\frac{1}{\varphi_{\text {major }}(-t)} .
$$

Если фиксировать значения интегралов $\mathscr{H}, \mathscr{T}$ и увеличивать значение $\mathscr{M}$, или, что то же самое, параметра $\kappa$, то траектории периодических решений $\varphi_{\text {major }} u \varphi_{\text {minor }}$ будут приближаться друг $\kappa$ другу и при некотором значении $\kappa=\kappa_{*}$ таком, что

$$
\frac{\nu^{2} x_{2}^{3}}{4}<\kappa_{*}<\frac{\nu^{2} x_{3}^{3}}{4},
$$

эти решения совпадают, т.е. при этом значении $\kappa_{*}$ на инвариантном торе будет только одно периодическое решение, а остальные будут асимптотически приближаться к нему.

Дальнейшее увеличение параметра к при неизменных значениях $\mathscr{H}, \mathscr{T}$ приводит к квазипериодическим и, в частности, к периодическим решениям $\varphi$. 
Решения системы дифференииальных уравнений (2.1) являются квазипериодическим для почти всех значений к, если

$$
\left|\nu x_{3}^{3 / 2}\right| \leqslant 2 \kappa
$$

при этом множество периодических решений имеет меру нуль.

ДокАЗАтЕльство. Пусть вначале $\sqrt{\mathscr{F}}>0$. Тогда для достаточно больших по абсолютной величине значений $\varphi$ функция $\varphi(t)$ убывает. Но если для любого значения $\mathscr{F}$ область $\dot{\varphi}>0$ не пуста, то никакая траектория решения, в которой $\varphi(t)$ убывает от $+\infty$, не может опуститься ниже этой области. Но тогда должна существовать предельная периодическая траектория.

Аналогично, существует предельная периодическая траектория с возрастанием $\varphi(t)$ от $-\infty$ при убывании времени.

Наконец, еще две периодические траектории получаются при $\sqrt{\mathscr{F}}<0$.

Получим условия существования области

$$
\dot{\varphi}=\nu \mathscr{F} \varphi-\frac{\kappa}{\mathscr{F}}\left(1+\varphi^{2}\right)>0 .
$$

Решение этого неравенства имеет вид

$$
\frac{\nu \mathscr{F}^{3 / 2}-\sqrt{\nu^{2} \mathscr{F}^{3}-4 \kappa^{2}}}{2 \kappa} \leqslant \varphi \leqslant \frac{\nu \mathscr{F}^{3 / 2}+\sqrt{\nu^{2} \mathscr{F}^{3}-4 \kappa^{2}}}{2 \kappa},
$$

и очевидно, что искомая область будет существовать, если неравенство

$$
\nu^{2} \mathscr{F}^{3}-4 \kappa^{2} \geqslant 0
$$

выполнено для всех $\mathscr{F}$, т.е. $\nu^{2} \mathscr{F}_{\min }^{3}-4 \kappa^{2} \geqslant 0$.

Если неравенство (7.5) не имеет вещественных решений, то $\nu^{2} \mathscr{F}_{\max }^{3}-4 \kappa^{2} \leqslant 0$, $\varphi$ монотонно убывает, что и приводит к квазипериодичности $\varphi$.

Оценки (7.1) и (7.2) следуют из очевидного факта, что значения $\varphi$ решений $\varphi_{\text {major }}, \varphi_{\text {minor }}$ не могут достигать таких значений, при которых $\dot{\varphi}$ отрицательны при всех возможных значениях $\mathscr{F}$, т.е. при условии $x_{2} \leqslant \mathscr{F} \leqslant x_{3}$.

Легко видеть, что верхняя оценка периодического решения $\varphi_{\text {major }}$ обратна нижней оценке решения $\varphi_{\text {minor }}$, а также что верхняя оценка периодического решения $\varphi_{\text {minor }}$ обратна нижней оценке решения $\varphi_{\text {major }}$. Это обстоятельство приводит к предположению

$$
\varphi_{\text {minor }}(t)=\frac{1}{\varphi_{\text {major }}(-t)},
$$

которое легко проверяется:

$$
\left(\frac{1}{\varphi}\right)^{\cdot}=-\frac{\dot{\varphi}}{\varphi^{2}}=-\frac{\nu \sqrt{\mathscr{F}} \varphi-\frac{\kappa}{\mathscr{F}}\left(1+\varphi^{2}\right)}{\varphi^{2}}=-\left(\nu \sqrt{\mathscr{F}}\left(\frac{1}{\varphi}\right)-\frac{\kappa}{\mathscr{F}}\left(1+\left(\frac{1}{\varphi}\right)^{2}\right)\right),
$$

при этом производную $\mathscr{F}$ надо брать со знаком минус перед корнем:

$$
A_{3} \dot{\mathscr{F}}=-\sqrt{2 A_{3} \mathscr{T} \mathscr{R} \mathscr{F}-2 A_{3} \mathscr{F}(\mathscr{F}-\mathscr{H})^{2}-\mathscr{M}^{2} \mathscr{R}}=-A_{3} \sqrt{\mathscr{P}_{3}(\mathscr{F})} .
$$


Полученное соотношение, как и его доказательство, весьма близко к сотношению $\varphi_{-}=-1 / \varphi_{+}$из предложения 1 для решений на разных листах функции $\sqrt{\mathscr{F}}$, однако по существу эти похожие формулы имеют совершенно различный контекст.

Если решения $\varphi(t),-\varphi(t)^{-1}$ в классических координатах задают одно и то же решение, то решения $\varphi(t), \varphi(-t)^{-1}$, вообще говоря, разные. Исключение составляет только тот случай, когда два предельных периодических решения склеиваются в одно.

Как было отмечено в замечании 4, движение в задаче Гесса происходит на двумерном вещественом торе. Вообще говоря, при непрерывном изменении параметров задачи отношение частот этого движения

$$
\omega=\lim _{t \rightarrow \infty} \frac{m(t)}{n(t)}
$$

будет изменяться $(m(t), n(t)$ - число полных оборотов вдоль базисных циклов). При иррациональном значении $\omega$ все траектории являются эргодическими, т.е. задаются почти периодическими функциями, а при рациональном существуют периодические, но могут быть, вообе говоря, и асимптотически периодические решения (см. [25]).

В рассматриваемой задаче Гесса ситуация является существенно более простой в силу линейности уравнений (2.8), получаемых из уравнений Риккати. Степени линейного отображения с мнимыми собственными значениями задают либо периодическую, либо квазипериодическую (см. [19]) последовательности отображений. Этот же результат следует из рассмотрения параметрического отображения последования (см. (5.5)).

ЗАмечАниЕ 13. Асимптотики (2.12) и (2.11) при $c=0$ не являются асимптотиками общего положения, поскольку функция $\varphi$ в окрестности особой точки $\mathscr{F}=\infty$ является локально однозначной только в этих случаях, а в остальных случаях является бесконечнозначной.

При этом если рассматривать задачу Гесса не просто с комплексным временем, а на прямом произведении $S_{2} \times \mathbb{C} P^{1}$ (см. замечание 4$)$, то периодическое решение будет однозначным на вещественной окружности, а непериодическое бесконечнозначным. Такой подход выглядит естественным, поскольку и решения общего положения являются бесконечнозначными.

Следовательно, вполне естественно предположить, что два особых однозначных в окрестности особой точки $\mathscr{F}=\infty$ решения как раз и есть те самые два периодических решения, о которых идет речь в теореме 3 .

Однако если связь между рассмотренными решениями и существует, то она, по-видимому, весьма нетривиальна, поскольку при подходящих указанных в теореме 3 условиях все вещественные решения могут быть квазипериодическими, а значит, периодических решений не будет вообще, но однозначные в окрестности точки $\mathscr{F}=\infty$ все-таки будут. 


\section{§ 8. Представление предельных периодических решений}

При описании решений задачи Гесса, несомненно, одним из важнейших является представление предельных периодических решений $\varphi_{\text {major }}, \varphi_{\text {minor }}$, тем более, что, зная эти представления, можно получить и все остальные в силу известного свойства уравнения Риккати.

Естественно искать эти представления как функцию от периодической функции $\mathscr{F}$.

График периодических функций $\varphi(\mathscr{F})$ на отрезке $\left[x_{2}, x_{3}\right]$ имеет вид эллипса, касающегося вертикальных прямых $x=x_{2}, x=x_{3}$, поскольку в силу дифференциального уравнения

$$
\varphi_{\mathscr{F}}^{\prime}=\frac{\nu \sqrt{\mathscr{F}} \varphi-\frac{\kappa}{\mathscr{F}}\left(1+\varphi^{2}\right)}{\dot{\mathscr{F}}}=\frac{\nu \mathscr{F}^{3 / 2} \varphi-\kappa\left(1+\varphi^{2}\right)}{\sqrt{\mathscr{P}_{2}(\mathscr{F}) \mathscr{F}}}
$$

в точках $x_{2}, x_{3}$ производная $\varphi_{\mathscr{F}}^{\prime}$ бесконечна. При этом график возрастает на области (см. (7.4))

$$
\nu \mathscr{F} \varphi-\frac{\kappa}{\mathscr{F}}\left(1+\varphi^{2}\right)>0
$$

убывает вне ее и, естественно, пересекает ее границу в точках с экстремальными значениями.

Достаточно грубым приближением графиков периодических функций $\varphi(\mathscr{F})$ является график функции $a_{0}+a_{1} \sqrt{\mathscr{F}}+a_{3} \dot{\mathscr{F}}$, поэтому естественно искать представление функций $\varphi(\mathscr{F})$ в виде, аналогичном представлению двоякопериодических решений через ю-функцию Вейерштрасса (см. [18]), а именно в виде

$$
\varphi(\mathscr{F})=\sqrt{\mathscr{F}} \Phi(\mathscr{F})+\dot{\mathscr{F}} \Psi(\mathscr{F}) .
$$

Подставив это представление в дифференциальное уравнение (2.8) для $\varphi$, получим

$$
\left\{\begin{array}{l}
\Phi^{\prime}=-\frac{\Phi}{2 \mathscr{F}}+\nu \Psi-\frac{2 \kappa \Phi \Psi}{\mathscr{F}}, \\
\mathscr{P}_{3} \Psi^{\prime}=-\mathscr{P}_{2} \Psi+\nu \mathscr{F} \Phi-\frac{\kappa\left(1+\mathscr{F} \Phi^{2}+\mathscr{P}_{3} \Psi^{2}\right)}{\mathscr{F}},
\end{array}\right.
$$

где $\Phi^{\prime}, \Psi^{\prime}$ - производные по $\mathscr{F}, \mathscr{P}_{3}=(\dot{\mathscr{F}})^{2}$ есть полином третьей степени по $\mathscr{F}$ (см. $(2.5)), \mathscr{P}_{2}=\ddot{\mathscr{F}}=\left(\mathscr{P}_{3}\right)^{\prime} / 2-$ полином второй степени по $\mathscr{F}$.

ПредлОжЕНИЕ 10. Функиия $\varphi(t)$, имеющая представление (8.1) и удовлетворяющая системе дифференииальных уравнений (8.2), является решением (2.8) как на листе $\sqrt{\mathscr{F}}$, так и на листе $-\sqrt{\mathscr{F}}$, т.е. задает глобальное решение (2.8).

Истинность предложения 10 следует из того, что дифференциальные уравнения (8.2) являются инвариантными относительно замены $\Phi \rightarrow-\Phi, \nu \rightarrow-\nu$.

ПреДлОжЕНИЕ 11. Система дифференииальных уравнений (8.2) имеет первый интеграл

$$
\mathscr{P}_{3} \Psi^{2}-\mathscr{F} \Phi^{2}+1=0
$$


что позволяет записать систему (8.2) в виде

$$
\left\{\begin{array}{l}
2 \mathscr{F} \Phi^{\prime}=-\Phi+2 \Psi(\nu \mathscr{F}-2 \kappa \Phi), \\
\mathscr{P}_{3} \Psi^{\prime}=-\mathscr{P}_{2} \Psi+\Phi(\nu \mathscr{F}-2 \kappa \Phi) .
\end{array}\right.
$$

ДокАЗАТЕЛьство. Убедиться в том, что $\mathscr{P}_{3} \Psi^{2}-\mathscr{F} \Phi^{2}$ является частным первым интегралом, можно простой проверкой:

$$
\left(\mathscr{P}_{3} \Psi^{2}-\mathscr{F} \Phi^{2}\right)^{\prime}=-\frac{2 \Psi \kappa}{\mathscr{F}}\left(\mathscr{P}_{3} \Psi^{2}-\mathscr{F} \Phi^{2}+1\right),
$$

но суть дела заключается в том, что решения $\varphi$ на разных листах функции $\sqrt{\mathscr{F}}$ связаны сотношением $\varphi_{+} \varphi_{-}=-1$ в силу предложения 1 , поэтому

$$
(\sqrt{\mathscr{F}} \Phi(\mathscr{F})+\dot{\mathscr{F}} \Psi(\mathscr{F}))(-\sqrt{\mathscr{F}} \Phi(\mathscr{F})+\dot{\mathscr{F}} \Psi(\mathscr{F}))+1=0 .
$$

Систему дифференциальных уравнений (8.4) можно записать в более симметричном виде. В самом деле,

$$
\left\{\begin{array}{l}
(\sqrt{\mathscr{F}} \Phi)^{\prime}=\frac{\Psi(\nu \mathscr{F}-2 \kappa \Phi)}{\sqrt{\mathscr{F}}}, \\
\left(\sqrt{\mathscr{P}_{3}} \Psi\right)^{\prime}=\frac{\Phi(\nu \mathscr{F}-2 \kappa \Phi)}{\sqrt{\mathscr{P}_{3}}},
\end{array}\right.
$$

и, полагая

$$
\widetilde{\Phi}=\sqrt{\mathscr{F}} \Phi, \quad \widetilde{\Psi}=\sqrt{\mathscr{P}_{3}} \Psi,
$$

получим

с первым интегралом

$$
\left\{\begin{array}{l}
\widetilde{\Phi}^{\prime}=\frac{\widetilde{\Psi}\left(\nu \mathscr{F}^{3 / 2}-2 \kappa \widetilde{\Phi}\right)}{\mathscr{F} \sqrt{\mathscr{P}_{3}}}, \\
\widetilde{\Psi}^{\prime}=\frac{\widetilde{\Phi}\left(\nu \mathscr{F}^{3 / 2}-2 \kappa \widetilde{\Phi}\right)}{\mathscr{F} \sqrt{\mathscr{P}_{3}}}
\end{array}\right.
$$

$$
\widetilde{\Psi}^{2}-\widetilde{\Phi}^{2}+1=0
$$

В результате имеем

$$
\begin{aligned}
& \left\{\begin{array}{l}
\widetilde{\Phi}=\operatorname{sh}\left(\int \frac{\nu \mathscr{F}^{3 / 2}-2 \kappa \widetilde{\Phi}}{\mathscr{F} \sqrt{\mathscr{P}_{3}}} d \mathscr{F}\right), \\
\widetilde{\Psi}=\operatorname{ch}\left(\int \frac{\nu \mathscr{F}^{3 / 2}-2 \kappa \widetilde{\Phi}}{\mathscr{F} \sqrt{\mathscr{P}_{3}}} d \mathscr{F}\right),
\end{array}\right. \\
& \widetilde{\Phi}=\frac{1}{2}\left(\varphi-\frac{1}{\varphi}\right), \quad \widetilde{\Psi}=\frac{1}{2}\left(\varphi+\frac{1}{\varphi}\right) .
\end{aligned}
$$

Полученные представления (8.4), (8.5) и (8.7), несмотря на их внешнюю привлекательность, вряд ли возможно использовать для для представления $\varphi$ в виде ряда по $\mathscr{F}$, поскольку в концах интересующего нас отрезка $\left[x_{2}, x_{3}\right]$ решение (8.3), (8.4) имеет логарифмические особенности. 
Можно вернуться к рассмотрению системы (8.2) и потребовать, чтобы правая часть второго уравнения системы в одной из точек $x_{2}, x_{3}$ равнялась нулю. Тогда мы получим представление без особенности в этой точке и зависимость между $\Phi\left(x_{i}\right)$ и $\Psi\left(x_{i}\right)$, но в таком случае не ясно, при каком значении, например, $\Phi\left(x_{2}\right)$ обеспечивается отсутствие особенности $\Phi(\mathscr{F})$ в точке $x_{3}$.

В создавшейся ситуации приходится в качестве аргумента функции $\varphi$ брать функцию $\dot{\mathscr{F}}$; тогда дифференциальное уравнение для $\varphi(\dot{\mathscr{F}})$ имеет вид

$$
\varphi^{\prime}=\frac{\nu \sqrt{\mathscr{F}} \varphi-\frac{\kappa}{\mathscr{F}}\left(1+\varphi^{2}\right)}{\ddot{\mathscr{F}}}=\frac{\nu \mathscr{F}^{3 / 2} \varphi-\kappa\left(1+\varphi^{2}\right)}{\mathscr{P}_{2}(\mathscr{F}) \mathscr{F}},
$$

где (см. (2.5))

$$
\mathscr{P}_{2}(\mathscr{F})=\frac{1}{2} \mathscr{P}_{3}(\mathscr{F})^{\prime}=2 A_{3}\left(-3 \mathscr{F}^{2}+4 \mathscr{F} \mathscr{H}+\mathscr{R} \mathscr{T}-\mathscr{H}^{2}\right) .
$$

Аналитическая функция $\varphi(\dot{\mathscr{F}})$ имеет особенности в точках, в которых

$$
\mathscr{F}_{0}=\frac{1}{3}\left(2 \mathscr{H} \pm \sqrt{\mathscr{H}^{2}+3 \mathscr{R} \mathscr{T}}\right) .
$$

Большая из этих точек попадает в отрезок $\left[x_{2}, x_{3}\right]$, и в этой точке нет особенности лишь в случае, когда числитель (2.5) равен нулю, т.е. при условии

$$
\varphi_{0}=\frac{\nu \mathscr{F}_{0}^{3 / 2} \pm \sqrt{\nu^{2} \mathscr{F}_{0}^{3}-4 \kappa^{2}}}{2 \kappa} .
$$

Как и следовало ожидать, искомые точки удовлетворяют соотношениям (7.1), (7.2), (7.4) и при этом $\varphi_{0+} \varphi_{0-}=1$.

Итак, представление периодических функций мы получаем в виде ряда Тейлора и к тому же сразу получаем точное условие, при котором предельные периодические решения случая Гесса склеиваются в одно, т.е. условие, разделяющее классы квазипериодических и периодических в пределе решений.

Теорема 4. Вещественные траектории решений в случае Гесса (см. теорему 1) задачи о движении тяжелого твердого тела на двумерном инвариантном торе, задаваемом первыми интегралами $\mathscr{H}, \mathscr{M}, \mathscr{T}, \mathscr{I}$, являются квазипериодическими, в частности, периодическими при условии

$$
\nu^{2} \mathscr{F}_{0}^{3}<4 \kappa^{2}, \quad \mathscr{F}_{0}=\frac{1}{3}\left(2 \mathscr{H}+\sqrt{\mathscr{H}^{2}+3 \mathscr{R} \mathscr{T}}\right),
$$

или

$$
\frac{8 B_{23} B_{31}}{A_{1} A_{2}} \mathscr{F}_{0}^{3}<\frac{\mathscr{M}^{2} \mathscr{R}}{A_{3}} .
$$

В случае

$$
\frac{8 B_{23} B_{31}}{A_{1} A_{2}} \mathscr{F}_{0}^{3}=\frac{\mathscr{M}^{2} \mathscr{R}}{A_{3}}
$$

на торе будет ровно одно периодическое решение, являющееся предельным для всех остальных решений. 
Наконеч, если

$$
\frac{8 B_{23} B_{31}}{A_{1} A_{2}} \mathscr{F}_{0}^{3}>\frac{\mathscr{M}^{2} \mathscr{R}}{A_{3}},
$$

на торе будет ровно два периодических решения, являющихся предельными для всех остальных решений.

В этом случае периодические решения имеют представление

$$
\varphi(\dot{\mathscr{F}})=\sum_{i=0}^{\infty} a_{i}\left(\dot{\mathscr{F}}-\dot{\mathscr{F}}_{0}\right)^{i},
$$

где

$$
\begin{gathered}
A_{3} \dot{\mathscr{F}}_{0}=\sqrt{2 A_{3} \mathscr{T} \mathscr{R} \mathscr{F}-2 A_{3} \mathscr{F}_{0}\left(\mathscr{F}_{0}-\mathscr{H}\right)^{2}-\mathscr{M}^{2} \mathscr{R}}=A_{3} \sqrt{\mathscr{P}_{3}\left(\mathscr{F}_{0}\right)}, \\
a_{0}=\frac{\nu \mathscr{F}_{0}^{3 / 2} \pm \sqrt{\nu^{2} \mathscr{F}_{0}^{3}-4 \kappa^{2}}}{2 \kappa}, \\
a_{i}=\tilde{a}_{i}(\varphi, \mathscr{F}) \mid \mathscr{F}=\mathscr{F}_{0}, \varphi=a_{0}, \\
\tilde{a}_{1}(\varphi, \mathscr{F})=2\left(\nu \sqrt{\mathscr{F}} \varphi-\frac{\kappa}{\mathscr{F}}\left(1+\varphi^{2}\right)\right)\left(\frac{d \mathscr{P}_{3}(\mathscr{F})}{d \mathscr{F}}\right)^{-1}, \\
\tilde{a}_{i+1}(\varphi, \mathscr{F})=2 \dot{\tilde{a}}_{i}(\varphi, \mathscr{F})\left(\frac{d \mathscr{P}_{3}(\mathscr{F})}{d \mathscr{F}}\right)^{-1} .
\end{gathered}
$$

ЗАмЕчАниЕ 14. Точное условие трансформации вида траекторий решений задачи Гесса от квазипериодических к предельно-периодическим получено как в терминах параметров $\mathscr{H}, \mathscr{M}, \mathscr{T}$ (теорема 4), так и в терминах параметров $a_{11}$, $a_{22}$ (см. (5.11), (5.13)), что дает возможность исследовать связь этих наборов параметров, которая, по-видимому, является весьма нетривиальной.

\section{Список литературы}

[1] W. Hess, "Ueber die Euler'schen Bewegungsgleichungen und über eine neue partikuläre Lösung des Problems der Bewegung eines starren Körpers um einen festen Punkt", Math. Ann., 37:2 (1890), 153-181.

[2] Н.Е. Жуковский, "Локсодромический маятник Гесса", Труды отд. физ. наук об-ва любит. естествознания, 5:2 (1893), 37-45.

[3] Б. К. Млодзеевский, П. А. Некрасов, "Об условиях существования асимптотических периодических движений в задаче Гесса", Труды отд. физ. наук об-ва любит. естествознания, 6:1 (1893), 43-52.

[4] П. А. Некрасов, “Аналитическое исследование одного случая движения тяжелого твердого тела около неподвижной точки”, Матем. сб., 18:2 (1896), 161-274.

[5] Г. Г. Аппельрот, "По поводу $\S 1$ мемуара С. В. Ковалевской «Sur le problème de la rotation d'un corps solide autour d'un point fixe»", Матем. сб., 16:3 (1892), 483-507.

[6] С. В. Ковалевская, Научные работьи, Изд-во АН СССР, М., 1948, 368 с.

[7] П. А. Некрасов, "К задаче о движении тяжелого твердого тела около неподвижной точки", Матем. сб., 16:3 (1892), 508-517. 
[8] В. Е. Захаров, С. В. Манаков, С. П. Новиков, Л. П. Питаевский, Теория солитонов. Метод обратной задачи, Наука, М., 1980, 320 с.; англ. пер.: S. Novikov, S. V. Manakov, L.P. Pitaevskii, V.E. Zakharov, Theory of solitons. The inverse scattering method, Contemp. Soviet Math., Consultants Bureau [Plenum], New York, 1984, xi+276 pp.

[9] С.В. Манаков, “Замечание об интегрировании уравнений Эйлера динамики n-мерного твердого тела", Функи. анализ и его прил., 10:4 (1976), 93-94; англ. пер.: S. V. Manakov, "Note on the integration of Euler's equations of the dynamics of an n-dimensional rigid body", Funct. Anal. Appl., 10:4 (1976), 328-329.

[10] А. С. Мищенко, А.Т. Фоменко, "Уравнения Эйлера на конечномерных группах Ли", Изв. АН СССР. Сер. матем., 42:2 (1978), 396-415; англ. пер.: A. S. Mishchenko, A. T. Fomenko, "Euler equations on finite-dimensional Lie groups", Math. USSR-Izv., 12:2 (1978), 371-389.

[11] M. Adler, P. van Moerbeke, "Completely integrable systems, Euclidean Lie algebras, and curves", Adv. in Math., 38:3 (1980), 267-317.

[12] M. Adler, P. van Moerbeke, "Linearization of Hamiltonian system, Jacobi varieties, and represetation theory", Adv. in Math., 38:3 (1980), 318-379.

[13] V. Dragović, B. Gagić, "An L-A pair for the Hess-Apel'rot system and a new integrable case for the Euler-Poisson equations on so(4) $\times$ so(4)", Proc. Roy. Soc. Edinburgh Sect. A, 131:4 (2001), 845-855.

[14] P. Lubowiecki, H. Żołądek, "The Hess-Appelrot system. I. Invariant torus and its normal hyperbolicity", J. Geom. Mech., 4:4 (2012), 443-467.

[15] P. Lubowiecki, H. Żołądek, "The Hess-Appelrot system. II. Perturbation and limit cycles", J. Differential Equations, 252:2 (2012), 1701-1722.

[16] В.И. Арнольд, Математические методы классической механики, 3-е изд., Наука, М., 1989, 472 с.; англ. пер. 1-го изд.: V.I. Arnold, Mathematical methods of classical mechanics, Grad. Texts in Math., 60, Springer-Verlag, New York, 1978, $\mathrm{xvi}+462 \mathrm{pp}$.

[17] А.В. Беляев, “Аналитические свойства решений задачи о движении тяжелого твердого тела в случае Гесса", Укр. матем. вестник, 2:33 (2005), 297-317; англ. пер.: A. V. Belyaev, "Analytic properties of solutions of the Euler-Poisson equations in the Hess case", Ukr. Math. Bull., 2:2005, 301-321.

[18] А. Гурвиц, Р. Курант, Теория функиий, Мир, М., 1968, 648 с.; пер. с нем.: A. Hurwitz, Vorlesungen über allgemeine Funktionentheorie und elliptische Funktionen, Herausgegeben und ergänzt durch einen Abschnitt über geometrische Funktionentheorie von R. Courant. Mit einem Anhang von H. Röhrl, 4. Aufl., Grundlehren Math. Wiss., 3, Springer-Verlag, Berlin-New York, 1964, xiii+706 pp.

[19] Б. М. Левитан, В.В. Жиков, Почти-периодические функиии и дифференциальные уравнения, Изд-во Моск. ун-та, М., 1978, 204 с.; англ. пер.: В. M. Levitan, V.V. Zhikov, Almost periodic functions and differential equations, Cambridge Univ. Press, Cambridge-New York, 1982, xi+211 pp.

[20] А.В. Беляев, "Об асимптотике особых точек решений задачи о движении тяжелого $n$-мерного тела в случае Лагранжа", Матем. сб., 202:11 (2011), 55-74; англ. пер.: А. V. Belyaev, "Asymptotic behaviour of singular points of solutions of the problem of heavy $n$-dimensional body motion in the Lagrange case", Sb. Math., 202:11 (2011), 1617-1635.

[21] A. V. Belyaev, "On the full list of finite-valued solutions of the Euler-Poisson equations having four first integrals", Math. Nachr., 285:10 (2012), 1199-1229.

[22] А. И. Докшевич, Решения в конечном виде уравнений Эйлера-Пуассона, Наукова думка, Киев, 1992, 168 с. 
[23] А. М. Ляпунов, “Об одном свойстве дифференциальных уравнений задачи о движении тяжелого твердого тела, имеющего неподвижную точку”, Сообщ. Харъковск. матем. об-ва, 2 сер., 4 (1894), 123-140.

[24] Ю.А. Архангельский, Аналитическал динамика твердого тела, Наука, М., 1977, $328 \mathrm{c}$.

[25] И. Тамура, Топология слоений, Мир, М., 1979, 319 с.; пер. с японск.: I. Tamura, Yōsō no toporoj̄̄ (Topology of foliations), Sugaku Sensho, Iwanami Shoten, Tokyo, 1976, $238 \mathrm{pp}$.

\author{
Александр Владимирович Беляев \\ (Alexandr V. Belyaev) \\ Донецкий государственный университет \\ управления, Украина \\ E-mail: alex.vl.belyaev@yandex.ru
}

Поступила в редакцию 03.02.2014 и 12.07.2014 